SEVERAL RELATED MODELS FOR MULTILAYER SANDWICH PLATES

By

Scott W. Hansen

IMA Preprint Series \# 1966

( February 2004)

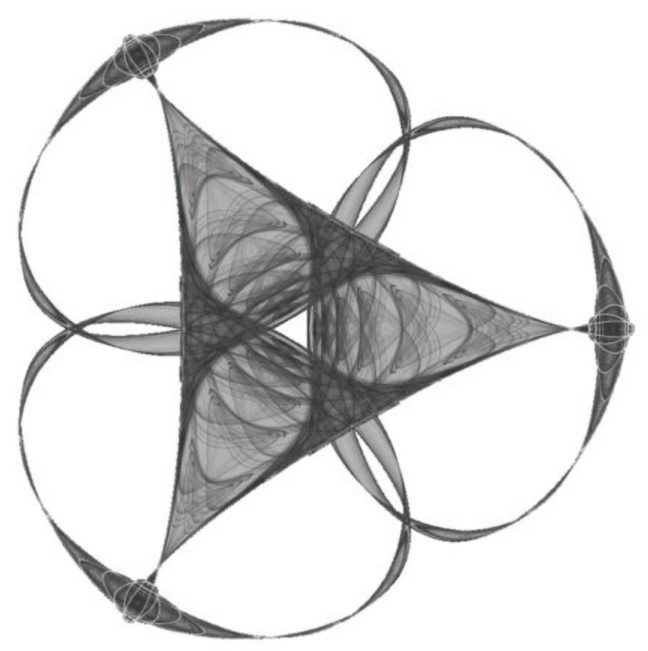

INSTITUTE FOR MATHEMATICS AND ITS APPLICATIONS

UNIVERSITY OF MINNESOTA

514 Vincent Hall

206 Church Street S.E.

Minneapolis, Minnesota 55455-0436

Phone: 612/624-6066 Fax: 612/626-7370

URL: http://www.ima.umn.edu 


\title{
SEVERAL RELATED MODELS FOR MULTILAYER SANDWICH PLATES
}

\author{
Scott W. Hansen \\ Iowa State University, \\ Ames, Iowa, 50011 USA \\ shanseniastate.edu
}

February 22, 2004

\begin{abstract}
Mathematical models for multilayer sandwich plates consisting of alternating stiff and compliant layers are derived. Two main types of models are described. First an initial model (analogous to the three-layer Rao-Nakra model) is derived under Kirchhoff plate assumptions for the stiff layers and Mindlin shear-deformable displacement assumptions for the compliant layers. The second type of model can be obtained from the original model by dropping the in-plane and rotational inertia. The resulting model is a generalization of the well-known model of Mead and Markus. Well-posedness and continuous parameter dependence results are described. Some variations of the initial model corresponding to thin compliant layers are described and shown to be regular perturbations of the initial model.
\end{abstract}

Keywords: Sandwich plate; multilayer plate; laminated plate.

AMS Subject Classification: 70H25, 74K20, 74M05

\section{Introduction}

Vibration of layered plate composites has been an area of considerable research over the past fifty years due to the importance of layered composites in a wide range of industrial and aerospace applications. Early on, it was found that plate models based upon the Kirchhoff hypothesis (that normal sections remain normal during deformation) gave acceptable predictions only for very thin plates where the compliances of the layers are comparable. (See Nosier and Reddy, [17] or Kapania and Raciti [10].) This is due to the fact that the effects of shear become significant when the compliances of the layers differ greatly. In fact, when a dissipative, compliant layer of an appropriate thickness is "sandwiched" between two relatively stiff layers, significant damping due to the shear motions in the compliant layer can be produced. This is often referred to as "constrained layer damping".

The first investigations of the mechanics of constrained layer damping were due to Ross, Unger, Kerwin [21] and Kerwin [9]. Their analysis of a sandwich beam consisting of two stiff 
face plates and a flexible core layer indicated that the dominant contribution to the flexural damping was due the shear (as opposed to extension) and moreover, an optimal level of shear damping in the compliant layer exists that leads to the best flexural damping (at a given frequency). Later, a number of three-layer sandwich beam and plate models were derived and the relationship between damping coefficients in the core layer and energy dissipation was studied in greater detail; e.g., DiTaranto [2], Mead and Markus [14], Yan and Dowell [23], Rao and Nakra [18], and others. (See Sun and Lu [22] for more background and further references.)

The sandwich model of Mead and Markus [14] and independently of DiTaranto [2] is perhaps the most well-known due to its simplicity and good agreement with experiment. In the one-dimensional case, this model (henceforth the "Mead-Markus model" in this paper) is based upon Euler-Bernoulli beam theory in the outer layers (ignoring longitudinal and rotational inertia), while the central core layer only includes transverse inertia and resistance to shear (i.e., longitudinal momentum, bending and extensional stresses are ignored in the core). The three layers are assumed to be bonded perfectly so that no slip occurs and the displacements are assumed to vary linearly in each layer, subject to the Kirchhoff hypothesis in the outer layers. Furthermore, as is standard in most beam and plate theories, the transverse normal stresses are assumed to be negligible.

The model of Rao and Nakra [18] is based upon similar displacement assumptions, however, all the inertial terms (in-plane and rotational) that are ignored in the Mead-Markus model are included. The core layer includes shear stresses, but bending stresses are assumed to be negligible. Rao and Nakra used a spectral analysis to compare their model with the Mead-Markus model. This analysis provided some indication of when (in terms of thicknesses, stiffnesses, frequencies, etc.,) it is necessary to include the rotational and translational inertia in the modeling.

In this paper we derive multilayer generalizations of the Rao-Nakra model and the MeadMarkus model consisting of alternating "stiff" and "compliant" layers. The multilayer RaoNakra model is derived under the same type of a-priori displacement assumptions from which the three-layer model is derived, namely Mindlin displacement assumptions for the compliant layers and Kirchhoff displacement assumptions for the stiff layers. However, in our formulation it is not necessary to neglect any of the membrane or bending stresses in the compliant layers. The variational equations of motion are obtained through application of Hamilton's principle. A multilayer generalization of the Mead-Markus model is then obtained from the multilayer Rao-Nakra model by omitting the dynamic terms that originate from rotational and translational kinetic energy. We show that the resulting system has a variational description. Existence and uniqueness results for each model are given.

One of the main goals of sandwich plate theory is to determine how damping in the compliant layer influences the dynamic behavior of the whole plate. In the above mentioned models for sandwich beams and plates, damping is treated by the so called "complex modulus" method (see e.g., Jones, [8]) which assumes a periodic solution (and hence ignores initial conditions). For the purposes of this paper, we assume a standard time-domain model for damping so that a boundary value problem can be studied. For simplicity the case of linear viscous damping in the compliant layers is assumed. (It is not difficult to adjust the system to allow for more general viscoelastic damping.) In the case of the multilayer Rao-Nakra model we show that damping due to shear in the compliant layers is mild enough so that 
the topology of the solution space is not changed.

In the modeling of layered plate systems, it is often unclear which forces can be safely ignored and which must be included in the model. As a step toward addressing this issue we prove a continuous parameter dependence result (Theorem 2.2) and identify two corresponding regular perturbations that result in a simpler form of the system of equations. The first, referred to as the "thin compliant layer model" is obtained by dropping all kinetic and force terms except those due to shear stresses in the compliant layers. That this is a regular perturbation lends justification to the modeling assumption used by Rao and Nakra [18], where the bending stresses were assumed to be negligible in the compliant layer. The second perturbation results in a model that can be viewed as a laminated plate model consisting of some number of layers bonded together by a thin adhesive. This model is obtained from the Rao-Nakra model by letting the thicknesses of the compliant layers tend to zero at a rate proportional to the shear parameter of those layers. Some special cases of the laminated plate with adhesive layers have been investigated in several papers [6], [5], [7].

We also mention that other approaches have been used to model multilayer beams and plates with multiple damping layers; some numerical models and models for beams are referenced in Sun and Lu [22] and an approach based on an analogy with classical threelayer theory is described in [8]. In addition there are a huge number of models for laminated plates which could potentially be used as a starting point for a multilayer sandwich plate model (see, e.g., surveys articles by Noor and Burton [16], and Kapania and Raciti [10]). Most of the laminate theories avoid treating each layer separately by averaging in various ways the stresses and elastic moduli through the depth. For example, classical laminate theory is based on Kirchhoff's theory for a single layer plate using an averaged cross-sectional stiffness; first-order shear deformation laminate theories are similarly based on the (singlelayer) Reissner [20], or Mindlin theory [15]. Higher-order theories have been developed that allow for quadratic and cubic behavior of the displacement as a function of the depth variable. In all of these approaches the number of equations is independent of the number of layers.

Laminate theories that treat each layer separately (the approach taken here) have been referred to as discrete layer theories. (See [16].) This approach has the advantage of retaining the geometry of the plate by allowing each layer to deform independent of other layers. Although these models are more complicated in the sense that the number of equations depends upon the number of layers, there is evidence that this approach leads to more accurate predictions than other laminate theories. (See Noor and Burton [16] for comparisons of several laminate theories to certain exact solutions.) Examples of dynamical discrete layer models include Hansen [4], Green and Naghdi [3], Reddy [20]. The main modeling difference here is that the Kirchhoff hypothesis is assumed to hold in the stiff layers due to the sandwich plate assumption that the compliances of alternate layers differ by several orders of magnitude.

This paper is organized as follows: In Section 2 we derive the multilayer Rao-Nakra plate model and prove existence, uniqueness and continuous parameter dependence of solutions. Some regular perturbations corresponding to thin adhesive layers of the initial model are described in Section 3. In Section 4 the multilayer Mead-Markus model is described. In Section 5 examples of some of the models described in this paper are written out in an explicit form for the case of a 3-layer plate. 


\section{Derivation of multilayer Rao-Nakra plate model}

In this section a multilayer version of the the Rao-Nakra sandwich plate model is derived. Well-posedness results are proved for the associated variational equations of motion. This model serves as a foundation for the derivation the other multilayer sandwich plate and laminated plate models described in this paper.

\subsection{Initial modeling assumptions}

The multilayer plate is assumed to consist of $n=2 m+1$ layers that occupy the region $\Omega \times(0, h)$ at equilibrium, where $\Omega$ is a smooth bounded domain in the plane. The total thickness $h$ is assumed to be small in comparison to the dimensions of $\Omega$. The plate is assumed to consist of alternating "stiff" and "compliant" plate layers, with stiff layers on the top and bottom. The layers are indexed from 1 to $n$, with odd indices for stiff layers and even indices for compliant layers.

It is assumed that (i) no slip occurs along the interfaces, (ii) within each layer the displacements vary linearly as a function of the transverse variable $x_{3}$, (iii) within each layer the transverse displacements are constant with respect to $x_{3}$, (iv) the Kirchhoff hypothesis applies to the stiff layers, i.e., normal sections remain normal during deformation.

Once the conservative equations of motion are obtained, the effects of damping can be included via the viscoelastic correspondence principle. Of particular interest is the case of damping due to shear in the compliant layers. (This is considered the dominant source of damping in most sandwich plate applications.)

Our derivation of the initial multilayer plate model is similar to the multilayer ReissnerMindlin model derived in Hansen, [4]. Here however, alternate layers are modeled under Kirchhoff plate assumptions.

Let

$$
0=z_{0}<z_{1}<\ldots<z_{n-1}<z_{n}=h, \quad h_{i}=z_{i}-z_{i-1}, i=1,2, \ldots, n
$$

We use the rectangular coordinates $x=\left\{x_{1}, x_{2}\right\}$ to denote points in $\Omega$ and $\left\{x, x_{3}\right\}$ to denote points in $Q=\cup_{i=1}^{n} Q_{i}$, where $Q_{i}$ is the reference configuration of the $i$ th layer given by

$$
Q_{i}=\Omega \times\left(z_{i-1}, z_{i}\right), \quad i=1,2, \ldots, n .
$$

For $\left\{x, x_{3}\right\} \in Q$ let $U\left(x, x_{3}\right)=\left\{U_{1}, U_{2}, U_{3}\right\}\left(x, x_{3}\right)$ denote the displacement vector (from reference configuration) and define $u^{i}=\left\{u_{1}^{i}, u_{2}^{i}\right\}$ and $u_{3}^{i}, i=0,1,2, \ldots n$ by $u_{j}^{i}(x)=U_{j}\left(x, z_{i}\right) \quad j=$ $1,2,3, \quad \forall x \in \Omega$.

Throughout this paper the index $i$ will refer to a particular layer or interface within the composite plate. For vector quantities whose components vary from layer to layer, the index $i$ will be superscripted, while for scalar quantities the $i$ will be subscripted.

Due to the assumption (iii) the transverse displacements $u_{3}^{i}(x)$ are independent of $i$, thus we may simply define the transverse displacement $w(x)$ as the scalar function $w(x)=$ $u_{3}^{i}(x)$, any $i, x \in \Omega$. Due to the assumptions (i) and (ii), the displacement $U\left(x, x_{3}\right)$ can be completely specified in terms of $w, u^{0}, \ldots, u^{n}$. For $i=1,2, \ldots, n$ define $\psi^{i}=\left\{\psi_{1}^{i}, \psi_{2}^{i}\right\}$, 


$$
\begin{aligned}
\varphi^{i}=\left\{\varphi_{1}^{i}, \varphi_{2}^{i}\right\}, \text { and } v^{i} & =\left\{v_{1}^{i}, v_{2}^{1}\right\} \text { by } \\
\psi^{i} & =\frac{u^{i}-u^{i-1}}{h_{i}}, \quad \varphi^{i}=\psi^{i}+\nabla w, \quad v^{i}=\frac{u^{i-1}+u^{i}}{2} .
\end{aligned}
$$

In addition, let $u, \psi, v, \varphi$ denote the matrices with $i$ th rows $u^{i}, \psi^{i}, v^{i}, \varphi^{i}$, respectively. The components $\psi_{j}^{i}$ of $\psi^{i}$ can be viewed as the total rotation angles (with negative orientation) of the deformed filament within the $i$-th layer in the $x_{j}-x_{3}$ plane. The components of $\varphi$ represent the (small angle approximation for the) shear angles within each layer. The components of $v^{i}$ represent the in-plane displacements of the midplanes of the $i$-th layer.

A notation is needed to indicate the even and odd indexed rows of vector and matrix quantities. Let $\psi_{E}$ denote the matrix consisting of the even indexed rows of $\psi$, i.e, $\psi_{E}^{i}=\psi^{2 i}$, for $i=1,2, \ldots m$. Analogously define the quantities $\varphi_{E}, v_{E}$. Likewise define $\psi_{O}, \varphi_{O}, v_{O}$, as the matrix consisting of the odd-indexed rows of $\psi, \varphi, v$. As further notation is developed, the same system regarding the subscripts $O$ and $E$ will be used.

Due to assumption (iv) regarding the Kirchhoff hypothesis, the odd components of $\varphi$ vanish. Thus from (2.1) we have the following equivalent equations:

$$
\varphi_{O}=0 ; \quad \psi_{O}=-\overrightarrow{1}_{O} \nabla w,
$$

where $\overrightarrow{1}_{O}$ denotes the $m+1$-vector of 1's. Similarly, with $\overrightarrow{1}_{E}$ denoting the $m$-vector of 1 's,

$$
\varphi_{E}=\psi_{E}+\overrightarrow{1}_{E} \nabla w
$$

Define $\hat{z}_{i}=\left(z_{i-1}+z_{i}\right) / 2$. Under Mindlin displacement assumptions [15], the displacement within the $i$ th layer can be written as

$$
\begin{array}{rlr}
U_{1}\left(x, x_{3}\right) & =v_{1}^{i}(x)+\left(x_{3}-\hat{z}_{i}\right) \psi_{1}^{i}(x) & z_{i-1}<x_{3}<z_{i} \\
U_{2}\left(x, x_{3}\right) & =v_{2}^{i}(x)+\left(x_{3}-\hat{z}_{i}\right) \psi_{2}^{i}(x) & z_{i-1}<x_{3}<z_{i} \\
U_{3}\left(x, x_{3}\right) & =w(x) & z_{i-1}<x_{3}<z_{i} .
\end{array}
$$

In the stiff layers ( $i$ odd) the above is adjusted by (2.2).

The displacement equations (2.2), (2.4) should be interpreted in terms of a minimal set of state variables. Note that due to (2.2) the in-plane displacements $U_{1}$ and $U_{2}$ within the stiff layers are completely determined by $v_{O}$ and $\nabla w$. This in turn completely determines the variables $u$, and consequently all other variables through the system of equations (2.1). Therefore $v_{O}$ and $w$ can be regarded as state variables and all other variables appearing in (2.4) can be expressed in terms of these.

Let $\sigma_{j k}, \epsilon_{j k}(j, k=1,2,3)$ denote the stress and strain tensors, respectively. Each layer is assumed to be homogeneous and transversely isotropic, however the material properties can vary from layer to layer. For the compliant layers we apply Mindlin constitutive assumptions, while for the stiff layers Kirchhoff displacement assumptions are applied.

The Mindlin stress-strain assumptions are

$$
\begin{array}{ll}
\sigma_{11}=\frac{E_{i}}{1-\nu_{i}^{2}}\left(\epsilon_{11}+\nu_{i} \epsilon_{22}\right) & \sigma_{12}=\frac{E_{i}}{1+\nu_{i}} \epsilon_{12} \\
\sigma_{22}=\frac{E_{i}}{1-\nu_{i}^{2}}\left(\nu_{i} \epsilon_{11}+\epsilon_{22}\right) & \sigma_{13}=2 G_{i} \epsilon_{13} \\
\sigma_{33}=0 & \sigma_{23}=2 G_{i} \epsilon_{23},
\end{array}
$$


where $E_{i}>0$ denotes the in-plane Young's modulus, $G_{i}>0$ denotes the transverse shear modulus, and $\nu_{i}$ denotes the in-plane Poisson's ratio $\left(0<\nu_{i}<1 / 2\right)$, all for the $i$ th layer.

For a small displacement theory it is assumed that

$$
\epsilon_{j k}(x)=\frac{1}{2}\left(\frac{\partial U_{j}(x)}{\partial x_{k}}+\frac{\partial U_{k}(x)}{\partial x_{j}}\right), \quad \forall x \in Q .
$$

Substituting (2.4) into (2.6) gives an expression for the strain within the $i$-th layer:

$$
\begin{aligned}
\epsilon_{11} & =\frac{\partial v_{1}^{i}}{\partial x_{1}}+\left(x_{3}-\hat{z}_{i}\right) \frac{\partial \psi_{1}^{i}}{\partial x_{1}} \quad \epsilon_{22}=\frac{\partial v_{2}^{i}}{\partial x_{2}}+\left(x_{3}-\hat{z}_{i}\right) \frac{\partial \psi_{2}^{i}}{\partial x_{2}} \\
\epsilon_{12} & =\frac{1}{2}\left[\frac{\partial v_{1}^{i}}{\partial x_{2}}+\frac{\partial v_{2}^{i}}{\partial x_{1}}+\left(x_{3}-\hat{z}_{i}\right)\left(\frac{\partial \psi_{1}^{i}}{\partial x_{2}}+\frac{\partial \psi_{2}^{i}}{\partial x_{1}}\right)\right] \\
\epsilon_{13} & =\frac{1}{2}\left(\varphi_{1}^{i}\right) \quad \epsilon_{23}=\frac{1}{2}\left(\varphi_{2}^{i}\right) .
\end{aligned}
$$

Since the stiff layers do not allow shear, (2.5) and (2.7) are adjusted in the odd layers by setting

$$
\epsilon_{13}=\epsilon_{23}=0 \text { in odd indexed layers. }
$$

\subsubsection{Expressions for potential and kinetic energy}

The strain energy $\mathcal{P}=\sum_{i=1}^{n} \mathcal{P}_{i}$ and kinetic energy $\mathcal{K}=\sum_{i=1}^{n} \mathcal{K}_{i}$ for the composite plate are given by

$$
\mathcal{P}_{i}=\frac{1}{2} \int_{Q_{i}} \sum_{j, k=1}^{3} \epsilon_{j k} \sigma_{j k} d x d x_{3}, \quad \mathcal{K}_{i}=\frac{1}{2} \int_{Q_{i}} \rho_{i}\left(\dot{U}_{1}^{2}+\dot{U}_{2}^{2}+\dot{U}_{3}^{2}\right) d x d x_{3}
$$

where $=d / d t$ and $\rho_{i}>0$ denotes the mass density per unit volume within the $i$-th layer.

From (2.5) and (2.7) the strain energy of the $i$ th layer can be written as

$$
\begin{gathered}
\mathcal{P}_{i}=\frac{h_{i}^{3}}{2} \int_{\Omega} D_{i}\left[\left(\frac{\partial \psi_{1}^{i}}{\partial x_{1}}\right)^{2}+\left(\frac{\partial \psi_{2}^{i}}{\partial x_{2}}\right)^{2}+2 \nu_{i}\left(\frac{\partial \psi_{2}^{i}}{\partial x_{2}} \frac{\partial \psi_{1}^{i}}{\partial x_{1}}\right)+\left(\frac{1-\nu_{i}}{2}\right)\left(\frac{\partial \psi_{1}^{i}}{\partial x_{2}}+\frac{\partial \psi_{2}^{i}}{\partial x_{1}}\right)^{2}\right] d x \\
+\frac{h_{i}}{2} \int_{\Omega} 12 D_{i}\left[\left(\frac{\partial v_{1}^{i}}{\partial x_{1}}\right)^{2}+\left(\frac{\partial v_{2}^{i}}{\partial x_{2}}\right)^{2}+2 \nu_{i}\left(\frac{\partial v_{1}^{i}}{\partial x_{1}} \frac{\partial v_{2}^{i}}{\partial x_{2}}\right)+\left(\frac{1-\nu_{i}}{2}\right)\left(\frac{\partial v_{1}^{i}}{\partial x_{2}}+\frac{\partial v_{2}^{i}}{\partial x_{1}}\right)^{2}\right] \\
+G_{i}\left(\left(\varphi_{1}^{i}\right)^{2}+\left(\varphi_{2}^{i}\right)^{2}\right) d x
\end{gathered}
$$

where $D_{i}=E_{i} / 12\left(1-\nu_{i}^{2}\right) . D_{i} h_{i}^{3}$ is the modulus of flexural rigidity for the $i$ th layer and $h_{i} G_{i}$ is the modulus of elasticity in shear for the $i$ th layer. For a stiff (odd-indexed) layer, the shear vanishes and hence the last term in (2.9) is absent and $\psi_{O}=-\overrightarrow{1}_{O} \nabla w$.

Likewise the kinetic energy of the $i$ th layer is

$$
\mathcal{K}_{i}=\frac{1}{2} \int_{\Omega} \rho_{i} h_{i}(\dot{w})^{2}+\frac{\rho_{i} h_{i}^{3}}{12}\left|\dot{\psi}^{i}\right|^{2}+\rho_{i} h_{i}\left|\dot{v}^{i}\right|^{2} d x
$$

Define the following $n$ by $n$ matrices:

$$
\begin{array}{ll}
\mathbf{h}=\operatorname{diag}\left(h_{1}, h_{2}, \ldots, h_{n}\right) & \mathbf{D}=\operatorname{diag}\left(D_{1}, D_{2}, \ldots, D_{n}\right) \\
\mathbf{p}=\operatorname{diag}\left(\rho_{1}, \rho_{2}, \ldots, \rho_{n}\right) & \mathbf{G}=\operatorname{diag}\left(G_{1}, G_{2}, \ldots, G_{n}\right) .
\end{array}
$$


If $\mathbf{K}$ represents a diagonal matrix, we let $\mathbf{K}_{O}$ and $\mathbf{K}_{E}$ represent the associated diagonal matrices of odd-indexed and even-indexed diagonal elements, respectively.

If $\theta$ and $\xi$ are matrices in $\mathbf{R}^{l m}$, by $\theta \cdot \xi$ we mean the scalar product in $\mathbf{R}^{l m}$. We also denote

$$
(\theta, \xi)_{\Omega}=\int_{\Omega} \theta \cdot \xi d x, \quad(\theta, \xi)_{\Gamma}=\int_{\Gamma} \theta \cdot \xi d \Gamma
$$

The expressions for the kinetic and potential energy can be rewritten as

$$
\mathcal{K}(t)=\tilde{c}(\dot{v}, \dot{\psi}, \dot{w} ; \dot{v}, \dot{\psi}, \dot{w}) / 2 \quad \mathcal{P}(t)=\tilde{a}(v, \psi, \varphi ; v, \psi, \varphi) / 2
$$

where $\tilde{c}(\cdot ; \cdot)$ and $\tilde{a}(\cdot ; \cdot)$ denote the bilinear forms

$$
\begin{aligned}
\tilde{c}(\psi, v, w ; \hat{\psi}, \hat{v}, \hat{w})= & ((\mathbf{h} \cdot \mathbf{p}) w, \hat{w})_{\Omega}+\left(\left(\mathbf{p h}^{3} / 12\right) \psi, \hat{\psi}\right)_{\Omega}+(\mathbf{h} \mathbf{p} v, \hat{v})_{\Omega} \\
\tilde{a}(\psi, v, \varphi ; \hat{\psi}, \hat{v}, \hat{\varphi})= & \ell\left(\mathbf{h}^{3} \mathbf{D} \psi ; \hat{\psi}\right)+12 \ell(\mathbf{h D} v ; \hat{v})+(\mathbf{G h} \varphi, \hat{\varphi})_{\Omega} \\
\ell(\psi, \hat{\psi}) & \sum_{i=1}^{n} \ell^{i}\left(\psi^{i} ; \hat{\psi}^{i}\right) \\
= & \left(\frac{\partial \psi_{1}^{i}}{\partial x_{1}}, \frac{\partial \hat{\psi}_{1}^{i}}{\partial x_{1}}\right)_{\Omega}+\left(\frac{\partial \psi_{2}^{i}}{\partial x_{2}}, \frac{\partial \hat{\psi}_{2}^{i}}{\partial x_{2}}\right)_{\Omega} \\
& \left.+\left(\nu_{i} \frac{\partial \psi_{2}^{i}}{\partial x_{2}}, \frac{\partial \hat{\psi}_{1}^{i}}{\partial x_{1}}\right)_{\Omega}+\left(\nu_{i} \frac{\partial \psi_{1}^{i}}{\partial x_{1}}, \frac{\partial \hat{\psi}_{2}^{i}}{\partial x_{2}}\right)_{\Omega} \hat{\psi}^{i}\right)=\left(\left(\frac{1-\nu_{i}}{2}\right)\left(\frac{\partial \psi_{1}^{i}}{\partial x_{2}}+\frac{\partial \psi_{2}^{i}}{\partial x_{1}}\right),\left(\frac{\partial \hat{\psi}_{1}^{i}}{\partial x_{2}}+\frac{\partial \hat{\psi}_{2}^{i}}{\partial x_{1}}\right)\right)_{\Omega} . \\
& +
\end{aligned}
$$

We can further decompose the energy as follows:

$$
\begin{aligned}
& \tilde{c}(\psi, v, w ; \hat{\psi}, \hat{v}, \hat{w})=((\mathbf{h} \cdot \mathbf{p}) w, \hat{w})_{\Omega}+c_{E}\left(\psi_{E}, v_{E} ; \hat{\psi}_{E}, \hat{v}_{E}\right)+c_{O}\left(\psi_{O}, v_{O} ; \hat{\psi}_{O}, \hat{v}_{O}\right) \\
& \tilde{a}(\psi, v, \varphi ; \hat{\psi}, \hat{v}, \hat{\varphi})=a_{E}\left(\psi_{E}, v_{E} ; \hat{\psi}_{E}, \hat{v}_{E}\right)+a_{O}\left(\psi_{O}, v_{O} ; \hat{\psi}_{O}, \hat{v}_{O}\right)+\left(\mathbf{G}_{E} \mathbf{h}_{E} \varphi_{E}, \hat{\varphi}_{E}\right)_{\Omega}
\end{aligned}
$$

where

$$
\begin{aligned}
& c_{E}\left(\psi_{E}, v_{E} ; \hat{\psi}_{E}, \hat{v}_{E}\right)=\left(\mathbf{p}_{E} \mathbf{h}_{E}^{3} \psi_{E}, \hat{\psi}_{E}\right)_{\Omega} / 12+\left(\mathbf{h}_{E} \mathbf{p}_{E} v_{E}, \hat{v}_{E}\right)_{\Omega} \\
& c_{O}\left(\psi_{O}, v_{O} ; \hat{\psi}_{O}, \hat{v}_{O}\right)=\left(\mathbf{p}_{O} \mathbf{h}_{O}^{3} \psi_{O}, \hat{\psi}_{O}\right)_{\Omega} / 12+\left(\mathbf{h}_{O} \mathbf{p}_{O} v_{O}, \hat{v}_{O}\right)_{\Omega} \\
& a_{O}\left(\psi_{O}, v_{O} ; \hat{\psi}_{O}, \hat{v}_{O}\right)=\ell_{O}\left(\mathbf{h}_{O}^{3} \mathbf{D}_{O} \psi_{O} ; \hat{\psi}_{O}\right)+12 \ell_{O}\left(\mathbf{h}_{O} \mathbf{D}_{O} v_{O} ; \hat{v}_{O}\right) \\
& a_{E}\left(\psi_{E}, v_{E} ; \hat{\psi}_{E}, \hat{v}_{E}\right)=\ell_{E}\left(\mathbf{h}_{E}^{3} \mathbf{D}_{E} \psi_{E} ; \hat{\psi}_{E}\right)+12 \ell_{E}\left(\mathbf{h}_{E} \mathbf{D}_{E} v_{E} ; \hat{v}_{E}\right)
\end{aligned}
$$

and the forms $\ell_{O}$ and $\ell_{E}$ are defined by the natural decomposition:

$$
\ell(\psi ; \hat{\psi})=\ell_{O}\left(\psi_{O} ; \hat{\psi}_{O}\right)+\ell_{E}\left(\psi_{E} ; \hat{\psi}_{E}\right)
$$

Using the relations $(2.1),(2.2),(2.4)$ one can solve for $v_{E}, \psi_{E}, \varphi_{E}$ in terms of $v_{O}, \nabla w$ :

$$
\begin{aligned}
v_{E} & =A v_{O}+\frac{1}{4} B \mathbf{h}_{O} \overrightarrow{1}_{O} \nabla w \\
\mathbf{h}_{E} \psi_{E} & =B v_{O}+A \mathbf{h}_{O} \overrightarrow{1}_{O} \nabla w \\
\varphi_{E} & =\psi_{E}+\overrightarrow{1}_{E} \nabla w=\mathbf{h}_{E}^{-1} B v_{O}+\vec{N} \nabla w
\end{aligned}
$$

where $\vec{N}=\mathbf{h}_{E}^{-1} A \mathbf{h}_{O} \overrightarrow{1}_{O}+\overrightarrow{1}_{E}$ and where $A=\left(a_{i j}\right), B=\left(b_{i j}\right)$ are the $m \times(m+1)$ matrices defined by

$$
a_{i j}=\left\{\begin{array}{ll}
1 / 2 & \text { if } j=i \text { or } j=i+1 \\
0 & \text { otherwise }
\end{array} \quad b_{i j}= \begin{cases}(-1)^{i+j+1} & \text { if } j=i \text { or } j=i+1 \\
0 & \text { otherwise }\end{cases}\right.
$$


Now define the forms $a$ and $c$ by

$$
\begin{aligned}
a\left(v_{O}, w ; \hat{v}_{O}, \hat{w}\right) & =\tilde{a}(\psi, v, \varphi ; \hat{\psi}, \hat{v}, \hat{\varphi}) \\
c\left(v_{O}, w ; \hat{v}_{O}, \hat{w}\right) & =\tilde{c}(\psi, v, w ; \hat{\psi}, \hat{v}, \hat{w})
\end{aligned}
$$

where the variables on the right hand side are expressed using (2.13) in terms of $v_{O}, w$ and $\hat{v}_{O}, \hat{w}$. For example, the form $c$ explicitly becomes

$$
\begin{aligned}
c\left(v_{O}, w ; \hat{v}_{O}, \hat{w}\right)=(\mathbf{p} \cdot \mathbf{h} w, \hat{w})_{\Omega}+c_{O}\left(-\overrightarrow{1}_{O} \nabla w, v_{O} ;-\overrightarrow{1}_{O} \nabla \hat{w}, \hat{v}_{O}\right)+ \\
c_{E}\left(\mathbf{h}_{E}^{-1}\left(B v_{O}+A \mathbf{h}_{O} \overrightarrow{1}_{O} \nabla w\right), A v_{O}+\frac{1}{4} B \mathbf{h}_{O} \overrightarrow{1}_{O} \nabla w ;\right. \\
\left.\mathbf{h}_{E}^{-1}\left(B \hat{v}_{O}+A \mathbf{h}_{O} \overrightarrow{1}_{O} \nabla \hat{w}\right), A \hat{v}_{O}+\frac{1}{4} B \mathbf{h}_{O} \overrightarrow{1}_{O} \nabla \hat{w}\right) \\
=(\mathbf{p} \cdot \mathbf{h} w, \hat{w})_{\Omega}+\left(\frac{1}{12} \mathbf{p}_{E} \mathbf{h}_{E}\left(B v_{O}+A \mathbf{h}_{O} \overrightarrow{1}_{O} \nabla w\right),\left(B \hat{v}_{O}+A \mathbf{h}_{O} \overrightarrow{1}_{O} \nabla \hat{w}\right)\right)_{\Omega} \\
\quad+\left(\mathbf{p}_{E} \mathbf{h}_{E}\left(A v_{O}+\frac{1}{4} B \mathbf{h}_{O} \overrightarrow{1}_{O} \nabla w\right),\left(A \hat{v}_{O}+\frac{1}{4} B \mathbf{h}_{O} \overrightarrow{1}_{O} \nabla \hat{w}\right)\right)_{\Omega} \\
\quad+\left(\frac{1}{12} \mathbf{p}_{O} \mathbf{h}_{O}\left(-\overrightarrow{1}_{O} \nabla w\right),\left(-\overrightarrow{1}_{O} \nabla \hat{w}\right)\right)_{\Omega}+\left(\mathbf{h}_{O} \mathbf{p}_{O} v_{O}, \hat{v}_{O}\right)_{\Omega} .
\end{aligned}
$$

\subsubsection{Applied forces, expression for work}

In order to write down an equation of motion some boundary conditions need to be specified. To set ideas we suppose that the plate is clamped on a portion of its boundary $\Gamma_{0} \subset \Gamma$ of positive measure. This means $w$, its normal derivative $\frac{\partial w}{\partial n}$ and $v$ are assumed to vanish on $\Gamma_{0}$. Let $\Gamma_{1}=\Gamma-\Gamma_{0}$ denote the free portion of the boundary.

Suppose the composite plate is subject to a volume distribution of forces $F=\left(\tilde{f}_{1}, \tilde{f}_{2}, \tilde{f}_{3}\right)$ and a surface distribution of forces $G=\left(\tilde{g}_{1}, \tilde{g}_{2}, \tilde{g}_{3}\right)$ along $\Gamma_{1}$. For consistency with the assumption that the transverse normal stresses vanish it is appropriate to assume that $\tilde{f}_{3}$ and $\tilde{g}_{3}$ are independent of $x_{3}$. Furthermore for consistency with the Kirchhoff hypothesis (i.e, that transverse shear strains vanish) it is appropriate to assume that $\tilde{f}_{1}$ and $\tilde{f}_{2}$ are independent of $x_{3}$ in the odd layers. Thus at the cost of possibly neglecting force moments within the even layers, we assume for simplicity that $F$ and $\tilde{g}_{3}$ are independent of $x_{3}$.

The work done on the plate by transverse forces is

$$
\mathcal{W}_{\text {trans }}=\int_{\Omega} w f_{3} d x+\int_{\Gamma_{1}} w g_{3} d \Gamma
$$

where (with $t$ suppressed) $f_{3}\left(x_{1}, x_{2}\right)=\int_{0}^{h} \tilde{f}_{3} d x_{3}$; and $g_{3}\left(x_{1}, x_{2}\right)=\int_{0}^{h} \tilde{g}_{3} d x_{3}$.

Next let $f^{i}\left(x_{1}, x_{2}\right), g^{i}\left(x_{1}, x_{2}\right)$ and $m^{i}\left(x_{1}, x_{2}\right)$ be the resultants

$$
f^{i}=\int_{z_{i-1}}^{z_{i}}\left(\tilde{f}_{1}, \tilde{f}_{2}\right) d x_{3}, \quad g^{i}=\int_{z_{i-1}}^{z_{i}}\left(\tilde{g}_{1}, \tilde{g}_{2}\right) d x_{3}, \quad m^{i}=\int_{z_{i-1}}^{z_{i}}\left(\tilde{g}_{1}, \tilde{g}_{2}\right)\left(x_{3}-\hat{z}_{i}\right) d x_{3} .
$$

Define $f$ and $g$ to be the matrices with $i$ th rows $f^{i}$ and $g^{i}$, respectively. In addition let $f_{O}, f_{E}$ denote the corresponding matrices containing only the odd and even rows of $f$, respectively. Define the other force matrices $g_{O}, g_{E}, m_{E}$ and $m_{O}$ in a likewise manner. 
The work due to in-plane forces within the odd layers is given by

$$
\mathcal{W}_{O}=\int_{\Omega} v_{O} \cdot f_{O} d x+\int_{\Gamma_{1}}-\overrightarrow{1}_{O} \nabla w \cdot m_{O}+v_{O} \cdot g_{O} d \Gamma
$$

A similar expression exists for the work on the even layers:

$$
\mathcal{W}_{E}=\int_{\Omega} v_{E} \cdot f_{E} d x+\int_{\Gamma_{1}} \psi_{E} \cdot m_{E}+v_{E} \cdot g_{E} d \Gamma
$$

However, using (2.13) $\mathcal{W}_{E}$ can be expressed in the same form as $\mathcal{W}_{O}$. Hence, by renaming $f_{O}, m_{O}$, and $g_{O}$, the contribution from $\mathcal{W}_{E}$ may be included in the expression for $\mathcal{W}_{O}$.

When the work due to transverse forces is included, the total work done by external forces is expressed as

$$
\begin{aligned}
\mathcal{W}\left(v_{O}, w\right)(t)= & \int_{\Omega} w f_{3}+v_{O} \cdot f_{O} d x+\int_{\Gamma_{1}} w g_{3}-\nabla_{O} w \cdot m+v_{O} \cdot g_{O} d s \\
= & \int_{\Omega} w f_{3}+v_{O} \cdot f_{O} d x+\int_{\Gamma_{1}} w g_{3}-w_{n} M_{n}-w_{\tau} M_{\tau}+v_{O} \cdot g_{O} d s \\
= & \left(f_{3}, w\right)_{\Omega}+\left(f_{O}, v_{O}\right)_{\Omega} \\
& +\int_{\Gamma_{1}} g_{O} v_{O}-M_{n} w_{n}+\left(g_{3}+\frac{\partial M_{\tau}}{\partial \tau}\right) w d s
\end{aligned}
$$

where

$$
M=\sum_{i \text { odd }} m^{i}, \quad M_{\tau}=M \cdot \vec{\tau}, \quad M_{n}=M \cdot \vec{n}
$$

with $\vec{n}=\left(n_{1}, n_{2}\right)$ being the normal vector to $\Gamma$ and $\vec{\tau}=\left(-n_{2}, n_{1}\right)$ the tangent vector. In the second line of (2.15) we have written $\nabla w=w_{n} \vec{n}+w_{\tau} \vec{\tau}$, (normal and tangential components) and have integrated $M_{\tau}$ by parts on $\Gamma_{1}$ to obtain the last equality in (2.18).

Let us redefine $g_{3}$ to include the term next to it in (2.15):

$$
\left[g_{3}\right]_{\text {new }}=\left[g_{3}+\frac{\partial M_{\tau}}{\partial \tau}\right]_{\text {old }}
$$

This way the total work done by external forces can be written as:

$$
\mathcal{W}\left(v_{O}, w\right)(t)=\left(f_{3}, w\right)_{\Omega}+\left(f_{O}, v_{O}\right)_{\Omega}+\int_{\Gamma_{1}} g_{O} v_{O}-M_{n} w_{n}+g_{3} w d s
$$

\subsubsection{Weak form of equations of motion}

The Lagrangian $\mathcal{L}$ on $(0, T)$ is defined by

$$
\begin{aligned}
\mathcal{L} & =\int_{0}^{T} \mathcal{K}(t)+\mathcal{W}(t)-\mathcal{P}(t) d t \\
& =\int_{0}^{T} \frac{1}{2}\left(c\left(\dot{v}_{O}, \dot{w} ; \dot{v}_{O}, \dot{w}\right)-a\left(v_{O}, w ; v_{O}, w\right)\right)+\mathcal{W}\left(v_{O}, w\right) d t
\end{aligned}
$$

According to the principle of virtual work, the solution trajectory is the trajectory which renders stationary the Lagrangian under all kinematically admissible displacements. 
Let $\left\{\hat{v}_{O}, \hat{w}\right\}$ denote a test function on $\Omega \times(0, T)$ (with dimensionality matching that of $\left.\left\{v_{O}, w\right\}\right)$ for which $\left\{\hat{v}_{O}, \hat{w}\right\}=\left\{\frac{\partial \hat{v}_{O}}{\partial n}, \frac{\partial \hat{w}}{\partial n}\right\}=0$ on $\Gamma_{0} \times(0, T)$ and

$$
\left.\left\{\hat{v}_{O}, \hat{w}\right\}\right|_{t=0}=\left.\frac{\partial}{\partial t}\left\{\hat{v}_{O}, \hat{w}\right\}\right|_{t=0}=\left.\left\{\hat{v}_{O}, \hat{w}\right\}\right|_{t=T}=\left.\frac{\partial}{\partial t}\left\{\hat{v}_{O}, \hat{w}\right\}\right|_{t=T}=0 \quad \text { in } \Omega
$$

where $n$ is the outward unit normal to $\Gamma$. We set

$$
0=\lim _{\epsilon \rightarrow 0} \frac{\mathcal{L}\left(\left(v_{O}, w\right)+\epsilon\left(\hat{v}_{O}, \hat{w}\right)\right)-\mathcal{L}\left(v_{O}, w\right)}{\epsilon}
$$

to obtain the equations of motion in weak form:

$$
\int_{0}^{T} c\left(\dot{v}_{O}, \dot{w} ; \dot{\hat{v}}_{O}, \dot{\hat{w}}\right)-a\left(v_{O}, w ; \hat{v}_{O}, \hat{w}\right)-\mathcal{W}\left(\hat{v}_{O}, \hat{w}\right) d t=0 .
$$

\subsection{Existence, uniqueness, continuous parameter dependence}

We first consider the variational formulation of the initial/boundary value problem.

Define the spaces

$$
\begin{aligned}
H_{\Gamma_{0}}^{1} & =\left\{\varphi: \varphi \in H^{1}(\Omega), \varphi=0 \text { on } \Gamma_{0}\right\} \\
H_{\Gamma_{0}}^{2} & =\left\{\varphi \in H_{\Gamma_{0}}^{1}: \frac{\partial}{\partial x_{i}} \varphi \in H_{\Gamma_{0}}^{1}, i=1,2\right\} \\
L_{O}^{2}(\Omega) & =\left\{v_{O}=\left(v_{j}^{i}\right), i=1,3,5, \ldots n, j=1,2: v_{j}^{i} \in L^{2}(\Omega)\right\} \\
H_{O, \Gamma_{0}}^{1} & =\left\{v_{O} \in L_{O}^{2}(\Omega): v_{j}^{i} \in H_{\Gamma_{0}}^{1}, v_{O}=0 \text { on } \Gamma_{0}\right\} \\
L_{E}^{2}(\Omega) & =\left\{\phi_{E}=\left(\phi_{j}^{i}\right), i=2,4, \ldots 2 m, j=1,2: \phi_{j}^{i} \in L^{2}(\Omega)\right\} \\
H_{E, \Gamma_{0}}^{1} & =\left\{\phi_{E} \in L_{E}^{2}(\Omega): \phi_{j}^{i} \in H_{\Gamma_{0}}^{1}, \phi_{E}=0 \text { on } \Gamma_{0}\right\} .
\end{aligned}
$$

The energy space is $\mathcal{V} \times \mathcal{H}$, where

$$
\mathcal{V}=\left\{\left\{v_{O}, w\right\} \in H_{O, \Gamma_{0}}^{1} \times H_{\Gamma_{0}}^{2}\right\}, \quad \mathcal{H}=L_{O}^{2}(\Omega) \times H_{\Gamma_{0}}^{1}
$$

The variational formulation of the IBVP (initial boundary value problem) is:

VP1: Find $y=\left\{v_{O}, w\right\}$ such that

$$
\begin{aligned}
& y \in C([0, T], \mathcal{V}) \cap C^{1}([0, T], \mathcal{H}) \\
& c(\ddot{y} ; \hat{y})+a(y ; \hat{y})=\mathcal{W}(\hat{y}) \quad \forall \hat{y}=\left\{\hat{v}_{O}, \hat{w}\right\} \in \mathcal{V}
\end{aligned}
$$

(in the sense of distributions on $(0, T)$ )

$$
\left.y\right|_{t=0}=y^{0} \text { given in } \mathcal{V},\left.\quad \dot{y}\right|_{t=0}=y^{1} \text { given in } \mathcal{H}
$$

The forces $\left\{f_{O}, f_{3}, g_{O}, g_{3}, M_{n}\right\}$ that define $\mathcal{W}$ are assumed to be in the class $L^{2}$ in time and space (on $(0, T) \times \Omega$ for $f_{O}, f_{3}$ and $(0, T) \times \Gamma$ for the others). The parameters that define the forms $a$ and $c$ are assumed to satisfy the following: the thicknesses $h_{i}$ and Poisson's ratios $\nu_{i}$ are assumed to be positive constants (with $\nu_{i}<1 / 2$ ), the densities $\rho_{i}$ and shear moduli $G_{i}$ are assumed to be continuous on $\bar{\Omega}$ and the stiffnesses $D_{i}$ are assumed to be $C^{1}$ on $\bar{\Omega}$ for $i=1, \ldots n$. For simplicity all coefficients are assumed to be time-independent.

Let $a(\cdot), c(\cdot)$ denote the (nonnegative) quadratic forms associated with the bilinear forms $a$ and $c$; e.g., $a(u, w)=a(u, w ; u, w)$. 
Lemma 2.1 Assume (in addition to the conditions described for VP1) that all coefficients appearing in the definition of the forms $a$ and $c$ are nonnegative and those corresponding to odd layers are positive. Then the bilinear forms $a(\cdot ; \cdot)$ and $c(\cdot ; \cdot)$ are symmetric and continuous on $\mathcal{V} \times \mathcal{V}$ and $\mathcal{H} \times \mathcal{H}$, respectively. Furthermore there exist $\delta>0$, independent of all coefficients within even layers, for which

$$
\begin{array}{ll}
a\left(v_{O}, w\right) \geq \delta\left\|\left\{v_{O}, w\right\}\right\|_{\mathcal{V}}^{2} & \forall\left\{v_{O}, w\right\} \in \mathcal{V} \\
c\left(v_{O}, w\right) \geq \delta\left\|\left\{v_{O}, w\right\}\right\|_{\mathcal{H}}^{2} & \forall\left\{v_{O}, w\right\} \in \mathcal{H} .
\end{array}
$$

Proof: It is easy to see that both bilinear forms $a$ and $c$ are symmetric. Furthermore $c(\cdot ; \cdot)$ is continuous on $\mathcal{H} \times \mathcal{H}$ since it is a composition of continuous functions. Likewise $a(\cdot ; \cdot)$ is easily seen to be continuous on $\mathcal{V} \times \mathcal{V}$. From inspection of (2.14) we see that

$$
\begin{aligned}
c\left(v_{O}, w\right) & \geq(\mathbf{h} \cdot \mathbf{p} w, w)_{\Omega}+\left(\mathbf{p}_{O} \mathbf{h}_{O}^{3} \overrightarrow{1}_{O} \nabla w, \overrightarrow{1}_{O} \nabla w\right)_{\Omega}+\left(\mathbf{p}_{O} \mathbf{h}_{O} v_{O}, v_{O}\right)_{\Omega} \\
& \geq \delta\left\{\|w\|_{H^{1}(\Omega)}^{2}+\left\|v_{O}\right\|_{L_{O}^{2}(\Omega)}^{2}\right\}=\delta\left\|\left\{v_{O}, w\right\}\right\|_{\mathcal{H}^{2}}^{2} .
\end{aligned}
$$

Thus the estimate for $c\left(v_{O}, w\right)$ is valid.

If $\psi^{i} \in H_{\Gamma_{0}}^{1}$, Poincaré's inequality together with Korn's inequality (see e.g., Lagnese and Lions [12]; pp. 44-47) implies the coercivity:

$$
\ell^{i}\left(\psi^{i} ; \psi^{i}\right) \geq C\left\|\psi^{i}\right\|_{H^{1}(\Omega)}^{2} \quad \forall \psi^{i} \in H_{\Gamma_{0}}^{1}
$$

where $C>0$ and $i$ represents an odd layer. It follows that (using a different $C>0$ )

$$
\ell_{O}\left(\psi_{O} ; \psi_{O}\right) \geq C\left\|\psi_{O}\right\|_{H_{O, \Gamma_{0}}^{1}}^{2} \quad \forall \psi_{O} \in H_{O, \Gamma_{0}}^{1} .
$$

Since $w$ and $\frac{\partial w}{\partial n}$ vanish on $\Gamma_{0}, \nabla w$ must also vanish on $\Gamma_{0}$. Hence Poincaré's inequality gives

$$
\ell_{O}\left(\overrightarrow{1}_{O} \nabla w ; \overrightarrow{1}_{O} \nabla w\right) \geq C\left\|\overrightarrow{1}_{O} \nabla w\right\|_{H_{O, \Gamma_{0}}^{1}}^{2} \geq C\|w\|_{H^{2}}^{2} \quad \forall w \in H_{\Gamma_{0}}^{2}
$$

where $C>0$. Thus for some $\delta_{1}>0$ and $\delta>0$

$$
\begin{aligned}
a\left(v_{O}, w\right) & \geq a_{O}\left(-\overrightarrow{1}_{O} \nabla w, v_{O}\right) \\
& =\ell_{O}\left(\mathbf{h}_{O}^{3} \mathbf{D}_{O} \overrightarrow{1}_{O} \nabla w ; \overrightarrow{1}_{O} \nabla w\right)+12 \ell_{O}\left(\mathbf{h}_{O} \mathbf{D}_{O} v_{O} ; v_{O}\right) \\
& =\ell_{O}\left(\mathbf{h}_{O}^{3 / 2} \mathbf{D}_{O}^{1 / 2} \overrightarrow{1}_{O} \nabla w ; \mathbf{h}_{O}^{3 / 2} \mathbf{D}_{O}^{1 / 2} \overrightarrow{1}_{O} \nabla w\right)+12 \ell_{O}\left(\mathbf{h}_{O}^{1 / 2} \mathbf{D}_{O}^{1 / 2} v_{O} ; \mathbf{h}_{O}^{1 / 2} \mathbf{D}_{O}^{1 / 2} v_{O}\right) \\
& \geq \delta_{1}\left\|\left\{\mathbf{D}_{O}^{1 / 2} \mathbf{h}_{O}^{1 / 2} v o, \mathbf{D}_{O}^{3 / 2} \mathbf{h}_{O}^{1 / 2} w\right\}\right\|_{\mathcal{V}}^{2} \\
& \geq \delta\|\{v o, w\}\|_{\mathcal{V}}^{2} .
\end{aligned}
$$

Thus the first inequality of the lemma also holds.

Lemma 2.2 Assume in addition to the hypothesis of Lemma 2.1 that the diagonal elements of $\mathbf{h}_{E}^{-1} \mathbf{G}_{E}$ lie in a fixed bounded set $Q \subset C\left(\bar{\Omega}, \mathbf{R}^{m}\right)$. Then there exist $M>0$, independent of all coefficients within even layers (but depending upon $Q$ ), for which

$$
\begin{array}{lll}
a(v, w) \leq M\|\{v, w\}\|_{\mathcal{V}}^{2} & \forall\{v, w\} \in \mathcal{V} \\
c(v, w) \leq M\|\{v, w\}\|_{\mathcal{H}}^{2} & \forall\{v, w\} \in \mathcal{H} .
\end{array}
$$


Proof: From inspection of the energy terms $c_{E}, c_{O}, a_{E}$ and $a_{O}$ and the expression for $\psi_{E}$ in (2.13) it is clear that the only possible difficulty in bounding the energies is when $\mathbf{h}_{E}$ becomes singular. All terms in $c_{E}, c_{O}, a_{E}$ and $a_{O}$ that contain a $\psi_{E}$ also have an $\mathbf{h}_{E}^{3}$ which is more than enough to prevent a problem as an even-indexed thickness in $\mathbf{h}_{E}$ tends to zero. The only other possible problem is the shear term $\left(\mathbf{G}_{E} \mathbf{h}_{E} \varphi_{E}, \hat{\varphi}_{E}\right)_{\Omega}$. By (2.13) we have

$$
\begin{aligned}
\varphi_{E} & =\psi_{E}+\overrightarrow{1}_{E} \nabla w \\
& =\mathbf{h}_{E}^{-1} B v_{O}+\mathbf{h}_{E}^{-1} A \mathbf{h}_{O} \overrightarrow{1}_{O} \nabla w+\overrightarrow{1}_{E} \nabla w
\end{aligned}
$$

Thus as $\mathbf{h}_{E}^{-1}$ becomes unbounded, so does $\varphi_{E}$. On the other hand, when $\mathbf{h}_{E}^{-1} \mathbf{G}_{E}$ remains bounded,

$$
\begin{aligned}
\left(\mathbf{G}_{E} \mathbf{h}_{E} \varphi_{E}, \varphi_{E}\right)_{\Omega} & =\left(\left(\mathbf{G}_{E} \mathbf{h}_{E}^{-1}\right) \mathbf{h}_{E} \varphi_{E}, \mathbf{h}_{E} \varphi_{E}\right)_{\Omega} \\
& \leq C\left(\left(v_{O}, v_{O}\right)_{\Omega}+(\nabla w, \nabla w)_{\Omega}\right) \\
& \leq C\left\|\left\{v_{O}, w\right\}\right\|_{\mathcal{V}}^{2}, \quad \forall\left\{v_{O}, w\right\} \in \mathcal{V}
\end{aligned}
$$

where again $C$ is a positive constant. This completes the proof.

Theorem 2.1 Assume the coefficients satisfy the hypothesis of Lemma 2.1. Then the variational problem VP1 is well-set; i.e, there is a unique $y=\left\{v_{O}, w\right\}$ that satisfies (2.20)-(2.22).

For simplicity the following result is stated for the case of constant coefficients in the even layers, but a similar result could be worded and proved the same way for coefficients with the regularity of Lemmas 2.1, 2.2.

Let $Q_{\alpha}$ denote the space of nonnegative parameters appearing as the diagonal elements of the matrices $\left\{\mathbf{h}_{E}, \mathbf{p}_{E}, \mathbf{G}_{E}, \mathbf{D}_{E}\right\} \subset \mathbf{R}^{4 \mathbf{m}}$, such that $\left\|\operatorname{diag}\left(\mathbf{h}_{E}^{-1} \mathbf{G}_{E}\right)\right\|_{\mathbf{R}^{\mathbf{m}}} \leq \alpha$.

Theorem 2.2 Assume the coefficients satisfy the hypothesis of Theorem 2.1 and for some $\alpha>0$ the even coefficients belong to $Q_{\alpha}$. Let $P_{\epsilon} \rightarrow P_{0}$ in $Q_{\alpha}$. Then given any $T>0$, for fixed initial conditions and no applied forces, the corresponding solutions $\left\{y^{\epsilon}, \dot{y}^{\epsilon}\right\}$ converge to a solution $\left\{y^{0}, \dot{y}^{0}\right\}$ to the variational problem with parameters $P_{0}$ in the space $L^{2}((0, T) ; \mathcal{V} \times \mathcal{H})$.

Proof: It is enough to show that solutions vary continuously with respect to a single parameter $\epsilon$ (e.g., $\epsilon:=D_{2}$ or $\epsilon=\rho_{4}$, etc.,) as $\epsilon \rightarrow 0$. For each $\epsilon \in[0,1]$ the corresponding variational problems are: Find $y^{\epsilon}=\left\{v_{O}^{\epsilon}, w^{\epsilon}\right\}$ such that

$$
\begin{aligned}
& y \in C([0, T], \mathcal{V}) \cap C^{1}([0, T], \mathcal{H}) \\
& c^{\epsilon}\left(\ddot{y}^{\epsilon} ; \hat{y}\right)+a^{\epsilon}\left(y^{\epsilon} ; \hat{y}\right)=0 \quad \forall \hat{y}=\left\{\hat{v}_{O}, \hat{w}\right\} \in \mathcal{V} \\
& \left.\left\{y^{\epsilon}, \dot{y}^{\epsilon}\right\}\right|_{t=0}=\left\{Y^{0}, Y^{1}\right\} \text { given in } \mathcal{V} \times \mathcal{H}
\end{aligned}
$$

Let $X$ denote the Hilbert space $L^{2}((0, T), \mathcal{V} \times \mathcal{H})$. For $\{\phi, \psi\}$ in $\mathcal{V} \times \mathcal{H}$ define $\|\{\phi, \psi\}\|_{E^{\epsilon}}^{2}=$ $a^{\epsilon}(\phi ; \phi)+c^{\epsilon}(\psi ; \psi)$. Due to the uniform estimates in Lemmas 2.1 and 2.2, for $\epsilon \in[0,1]$, the 
energy norm $\|\cdot\|_{E^{\epsilon}}$ is equivalent to $\|\cdot\|_{\mathcal{V} \times \mathcal{H}}$. Therefore, instead of the standard norm for $X$, we may define for $\{\phi, \dot{\phi}\} \in X$,

$$
\|\{\phi, \dot{\phi}\}\|_{X}=\left(\int_{0}^{T}\|\{\phi, \dot{\phi}\}\|_{E^{0}}^{2} d t\right)^{1 / 2} .
$$

Using conservation of energy and the previously mentioned equivalence of energy norms, the solutions satisfy

$$
\left\|\left\{y^{\epsilon}, \dot{y}^{\epsilon}\right\}\right\|_{X} \leq C\left\|\left\{Y^{0}, Y^{1}\right\}\right\|_{\mathcal{V} \times \mathcal{H}},
$$

where $C$ is independent of $\epsilon \in(0,1)$. Since $X$ is a Hilbert space, a weakly convergent subsequence can be extracted so that $\left\{y^{\epsilon_{k}}, \dot{y}^{\epsilon_{k}}\right\} \rightarrow\{\bar{y}, \dot{\bar{y}}\}$ (weakly) in $X$. The continuity of $c$ and $a$ in Lemma 2.1 implies that $\{\bar{y}, \dot{\bar{y}}\}$ are solutions of the limiting variational problem. Thus by uniqueness of solutions, $\bar{y}=y^{0}$. If one assumes that $\left\{y^{\epsilon}, \dot{y}^{\epsilon}\right\}$ does not converge weakly to $\left\{y^{0}, \dot{y}^{0}\right\}$ then there exists $\{W, \dot{W}\} \in X$ so that a subsequence $<\left\{y^{\epsilon_{j}}, \dot{y}^{\epsilon_{j}}\right\},\{W, \dot{W}\}>_{X} \rightarrow$ $K \neq<\{y, \dot{y}\},\{W, \dot{W}\}>_{X}$. But this is impossible since one can take a subsequence of this subsequence, if necessary, to obtain that $<\left\{y^{\epsilon_{j}}, \dot{y}^{\epsilon_{j}}\right\},\{W, \dot{W}\}>_{X} \rightarrow<\left\{y^{0}, \dot{y}^{0}\right\},\{W, \dot{W}\}>_{X}$. Thus $\left\{y^{\epsilon}, \dot{y}^{\epsilon}\right\}$ converges weakly to $\left\{y^{0}, \dot{y}^{0}\right\}$.

Let $E^{\epsilon}(t)=a^{\epsilon}\left(y^{\epsilon} ; y^{\epsilon}\right)+c^{\epsilon}\left(\dot{y}^{\epsilon} ; \dot{y}^{\epsilon}\right)$. From conservation of energy we know that for $\epsilon \in[0,1]$, $\int_{0}^{T} E^{\epsilon}(t) d t=T E^{\epsilon}(0)$. Furthermore

$$
E^{\epsilon}(0)=a^{\epsilon}\left(Y^{0} ; Y^{0}\right)+c^{\epsilon}\left(Y^{1} ; Y^{1}\right) \rightarrow a^{0}\left(Y^{0} ; Y^{0}\right)+c^{0}\left(Y^{1} ; Y^{1}\right)=E^{0}(0) .
$$

Hence it follows that

$$
\int_{0}^{T} a^{0}\left(y^{\epsilon} ; y^{\epsilon}\right)+c^{0}\left(\dot{y}^{\epsilon} ; \dot{y}^{\epsilon}\right) d t \rightarrow \int_{0}^{T} a^{0}\left(y^{0} ; y^{0}\right)+c^{0}\left(\dot{y}^{0} ; \dot{y}^{0}\right) d t, \quad \text { as } \epsilon \rightarrow 0 .
$$

Therefore $\left\|\left\{y^{\epsilon}, \dot{y}^{\epsilon}\right\}\right\|_{X} \rightarrow\left\|\left\{y^{0}, \dot{y}^{0}\right\}\right\|_{X}$ and hence the strong convergence follows.

\subsection{Inclusion of damping}

Damping may be introduced into any of the plate layers by replacing the stress-strain relation (2.2) by an appropriate dissipative constitutive law. In the case of strain-rate damping, the stresses depend not only on the strains, but also the strain rate, so that (2.5) is modified to

$$
\begin{array}{ll}
\sigma_{11}=\frac{E+\tilde{E} d / d t}{1-\nu^{2}}\left(\epsilon_{11}+\nu \epsilon_{22}\right) & \sigma_{12}=\frac{E+\tilde{E} d / d t}{1+\nu} \epsilon_{12} \\
\sigma_{22}=\frac{E+E{ }^{2} d / d t}{1-\nu^{2}}\left(\nu \epsilon_{11}+\epsilon_{22}\right) & \sigma_{13}=2\left(G+\tilde{G} \frac{d}{d t}\right) \epsilon_{13} \\
\sigma_{33}=0 & \sigma_{23}=2\left(G+\tilde{G} \frac{d}{d t}\right) \epsilon_{23} .
\end{array}
$$

where $\tilde{E}$ and $\tilde{G}$ may depend upon $i$ and are assumed to be nonnegative.

Of course, general viscoelastic could also be considered; see e.g., Lagnese and Lions [12].

By the viscoelastic correspondence principle (e.g., see [12]) the equations of motion are given by simply replacing $E$ and $G$ by $E+\tilde{E} d / d t$ and $G+\tilde{G} d / d t$, respectively. The variational equation of motion (2.21) is modified to

$$
\begin{aligned}
& c\left(\ddot{v}_{O}, \ddot{w} ; \hat{v}_{O}, \hat{w}\right)+b\left(\dot{v}_{O}, \dot{w} ; \hat{v}_{O}, \hat{w}\right)+a\left(v_{O}, w ; \hat{v}_{O}, \hat{w}\right) \\
= & \int_{\Omega} \hat{w} f_{3}+\hat{v}_{O} \cdot f_{O} d x+\int_{\Gamma_{1}} \hat{w} g_{3}-\hat{w}_{n} M_{n}+\hat{v}_{O} \cdot g_{O} d s
\end{aligned}
$$


where the form $b(\cdot ; \cdot)$ is defined in an identical fashion as the way $a(\cdot ; \cdot)$ was defined, however, with $E$ and $G$ replaced by $\tilde{E}$ and $\tilde{G}$. (Of course, since $\mathbf{D}$ and $\mathbf{G}$ are defined in terms of $E$ and $G$, one also has to replace $\mathbf{D}$ and $\mathbf{G}$ by appropriate matrices, say $\tilde{\mathbf{D}}$ and $\tilde{\mathbf{G}}$, that are defined accordingly.)

In order for the standard variational theory to apply, it is enough that the bilinear form $b(\cdot ; \cdot)$ be symmetric, nonnegative and continuous on $\mathcal{V} \times \mathcal{V}$. (See Lions and Magenes [13] or Dautray and Lions [1].) However, this is automatic when the correspondence principle is applied since $b$ satisfies the same estimates as $a$. Hence, given an initial condition of the form (2.22), there is a unique solution $y=\left\{v_{O}, w\right\}$ to (2.28) within an appropriate function space.

In the case of shear damping, $b$ in $(2.28)$ is defined by

$$
b\left(\left\{v_{O}, w\right\},\left\{\hat{v}_{O}, \hat{w}\right\}\right)=\left(\tilde{\mathbf{G}}_{E} \mathbf{h}_{E} \varphi_{E}, \hat{\varphi}_{E}\right)_{\Omega} \quad \forall\left\{\hat{v}_{O}, \hat{w}\right\} \in \mathcal{H},
$$

where $\varphi_{E}$ and $\hat{\varphi}_{E}$ are related to $\left\{v_{O}, w\right\}$ and $\left\{\hat{v}_{O}, \hat{w}\right\}$ (respectively) by (2.13).

Here it is easy to see that $b(\cdot ; \cdot)$ be symmetric, nonnegative and continuous on $\mathcal{H} \times \mathcal{H}$. (This would not be true of damping which includes the extensional damping terms $\tilde{E}$ in (2.27).) In this case the function space of well-posedness remains the same as the undamped case (see Dautray and Lions [1]). Therefore the following result holds.

Proposition 2.1 Given any $y^{0}=\left\{v_{O}^{0}, w^{0}\right\} \in \mathcal{V}$ and any $y^{1}=\left\{v_{O}^{1}, w^{1}\right\} \in \mathcal{H}$ there exists a unique variational solution $y=\left\{v_{O}, w\right\}$ to (2.28) such that $y(0)=y^{0}, \dot{y}(0)=y^{1}$. More precisely, the variational problem $\mathbf{V P 1}$, but with (2.21) replaced by the variational differential equation (2.28)-(2.29) is well set.

\section{Regular perturbations of initial model}

In this section two perturbations of the multilayer Rao-Nakra system VP1 are described. The first, which we call the thin compliant layer Rao-Nakra model is obtained by letting $\rho_{E}$ and $\mathbf{D}_{E}$ tend to zero in the bilinear forms $a_{E}$ and $c_{E}$. This approximation retains the potential energy of shear and transverse kinetic energy for the compliant layers. The second approximation can be viewed as a laminated plate with adjacent layers bonded together by an infinitesimally thin adhesive. We obtain this model (henceforth, the laminated plate with adhesive bonding, by letting $\mathbf{G}_{E}$ and $\mathbf{h}_{E}$ both tend to zero at a proportionate rate (in each layer the rate can be different) in the thin compliant layer Rao-Nakra model.

In order to write out the associated boundary value problems some notation will be needed. To keep thing simple, in this section it will be assumed that the stiffness $D_{i}$ and Poisson's ratios $\nu_{i}$ are constant (but can depend upon $i$ ). (This allows the strong form of the equations of motion to take a slightly simpler from.)

\subsection{Some notation for differential and boundary operators}

Define for $i=1,2, \ldots n$ and sufficiently smooth $\phi=\left\{\phi_{1}, \phi_{2}\right\}(x)$ the operators $\mathcal{M}^{i}[\phi]$ by

$$
\mathcal{M}^{i}[\phi]=\left(\begin{array}{cc}
\varepsilon_{11}+\nu_{i} \varepsilon_{22} & \left(1-\nu_{i}\right) \varepsilon_{12} \\
\left(1-\nu_{i}\right) \varepsilon_{12} & \nu_{i} \varepsilon_{11}+\varepsilon_{22}
\end{array}\right) ; \quad \varepsilon_{j k}(\phi)=\frac{1}{2}\left(\frac{\partial \phi_{j}}{\partial x_{k}}+\frac{\partial \phi_{k}}{\partial x_{j}}\right) .
$$


Thus $\mathcal{M}^{i}[\phi]$ is a symmetric matrix for every $x \in \Omega$.

We define the divergence of a symmetric matrix to be the divergence of each column:

$$
\operatorname{div}\left(\begin{array}{ll}
a_{11} & a_{12} \\
a_{12} & a_{22}
\end{array}\right)=\left\{\operatorname{div}\left(\begin{array}{l}
a_{11} \\
a_{12}
\end{array}\right), \operatorname{div}\left(\begin{array}{l}
a_{12} \\
a_{22}
\end{array}\right)\right\} .
$$

Then

$$
L^{i} \phi=\left\{L_{1}^{i} \phi, L_{2}^{i} \phi\right\}=\operatorname{div}\left(\mathcal{M}^{i}[\phi]\right)
$$

defines a second order operator which is given explicitly by

$$
\begin{aligned}
& L_{1}^{i} \phi=\frac{\partial}{\partial x_{1}}\left[\left(\frac{\partial \phi_{1}}{\partial x_{1}}+\nu_{i} \frac{\partial \phi_{2}}{\partial x_{2}}\right)\right]+\frac{\partial}{\partial x_{2}}\left[\left(\frac{1-\nu_{i}}{2}\right)\left(\frac{\partial \phi_{1}}{\partial x_{2}}+\frac{\partial \phi_{2}}{\partial x_{1}}\right)\right] \\
& L_{2}^{i} \phi=\frac{\partial}{\partial x_{2}}\left[\left(\frac{\partial \phi_{2}}{\partial x_{2}}+\nu_{i} \frac{\partial \phi_{1}}{\partial x_{1}}\right)\right]+\frac{\partial}{\partial x_{1}}\left[\left(\frac{1-\nu_{i}}{2}\right)\left(\frac{\partial \phi_{2}}{\partial x_{1}}+\frac{\partial \phi_{1}}{\partial x_{2}}\right)\right] .
\end{aligned}
$$

Also define the boundary operators $\mathcal{B}^{i} \phi=\left\{\mathcal{B}_{1}^{i}\left(\phi_{1}, \phi_{2}\right),\left(\mathcal{B}_{2}^{i}\left(\phi_{1}, \phi_{2}\right)\right\}\right.$ by

$$
\mathcal{B}^{i} \phi=\mathcal{M}^{i}[\phi] \vec{n},
$$

where $n=\left(n_{1}, n_{2}\right)$ denotes the outward unit normal to $\Gamma$. Explicitly one has

$$
\begin{aligned}
\mathcal{B}_{1}^{i}\left(\phi_{1}, \phi_{2}\right) & =\left[\left(\frac{\partial \phi_{1}}{\partial x_{1}} n_{1}+\nu_{i} \frac{\partial \phi_{2}}{\partial x_{2}} n_{1}\right)+\left(\frac{1-\nu_{i}}{2}\right)\left(\frac{\partial \phi_{1}}{\partial x_{2}}+\frac{\partial \phi_{2}}{\partial x_{1}}\right) n_{2}\right] \\
\mathcal{B}_{2}^{i}\left(\phi_{1}, \phi_{2}\right) & =\left[\left(\frac{\partial \phi_{2}}{\partial x_{2}} n_{2}+\nu_{i} \frac{\partial \phi_{1}}{\partial x_{1}} n_{2}\right)+\left(\frac{1-\nu_{i}}{2}\right)\left(\frac{\partial \phi_{2}}{\partial x_{1}}+\frac{\partial \phi_{1}}{\partial x_{2}}\right) n_{1}\right] .
\end{aligned}
$$

The following Green's formula is valid for all sufficiently smooth $\hat{\phi}, \phi$ :

$$
\ell^{i}(\phi, \hat{\phi})=\left(\mathcal{B}^{i} \phi, \hat{\phi}\right)_{\Gamma}-\left(L^{i} \phi, \hat{\phi}\right)_{\Omega}
$$

For $\xi=\left(\xi_{j}^{i}\right)(i=1,2, \ldots, n, j=1,2)$ define the matrices $L \xi$ and $\mathcal{B} \xi$ by

$$
(L \xi)_{i j}=\left(L_{j}^{i} \xi^{i}\right), \quad(\mathcal{B} \xi)_{i j}=\left(\mathcal{B}_{j}^{i} \xi^{i}\right), \quad i=1,2, \ldots, n, j=1,2 .
$$

Furthermore we define the operators $L_{O}, L_{E}, \mathcal{B}_{O}, \mathcal{B}_{E}$ from $L$ and $B$ based upon the convention that $O$ and $E$ subscripts refer to the parts of the operators that act upon odd and even rows respectively.

In particular, we will need Green's formulas for the expressions $\ell_{O}\left(\mathbf{h}_{O} \mathbf{D}_{O} v_{O}, \hat{v}_{O}\right)$ and $\ell_{O}\left(\mathbf{h}_{O}^{3} \mathbf{D}_{O} \overrightarrow{1}_{O} \nabla w, \overrightarrow{1}_{O} \nabla \hat{w}\right)$. In the first case, using (3.32) we have

$$
\ell_{O}\left(\mathbf{h}_{O} \mathbf{D}_{O} v_{O}, \hat{v}_{O}\right)=\left(\mathbf{h}_{O} \mathbf{D}_{O} \mathcal{B}_{O} v_{O}, \hat{v}_{O}\right)_{\Gamma}-\left(\mathbf{h}_{O} \mathbf{D}_{O} L_{O} v_{O}, \hat{v}_{O}\right) .
$$

In the latter case using (3.32) we obtain

$$
\begin{aligned}
\ell_{O}\left(\mathbf{h}_{O}^{3} \mathbf{D}_{O} \overrightarrow{1}_{O} \nabla w, \overrightarrow{1}_{O} \nabla \hat{w}\right)= & \left(\mathbf{D}_{O} \mathcal{B}_{O} \mathbf{h}_{O}^{3} \overrightarrow{1}_{O} \nabla w, \overrightarrow{1}_{O} \nabla \hat{w}\right)_{\Gamma_{1}}-\left(L_{O} \mathbf{h}_{O}^{3} \mathbf{D}_{O} \overrightarrow{1}_{O} \nabla w, \overrightarrow{1}_{O} \nabla \hat{w}\right)_{\Omega} \\
= & \left(\vec{n} \cdot \overrightarrow{1}_{O}^{T} \mathbf{D}_{O} \mathcal{B}_{O} \mathbf{h}_{O}^{3} \overrightarrow{1}_{O} \nabla w, \hat{w}_{n}\right)_{\Gamma_{1}}+\left(\operatorname{div} \overrightarrow{1}_{O}^{T} L_{O} \mathbf{h}_{O}^{3} \mathbf{D}_{O} \overrightarrow{1}_{O} \nabla w, \hat{w}\right)_{\Omega} \\
& -\left(\frac{\partial}{\partial \tau}\left\{\vec{\tau} \cdot \overrightarrow{1}_{O}^{T} \mathbf{D}_{O} \mathcal{B}_{O} \mathbf{h}_{O}^{3} \overrightarrow{1}_{O} \nabla w\right\}+\left(\overrightarrow{1}_{O}^{T} L_{O} \mathbf{h}_{O}^{3} \mathbf{D}_{O} \overrightarrow{1}_{O} \nabla w\right) \cdot \vec{n}, \hat{w}\right)_{\Gamma_{1}}
\end{aligned}
$$

where $\vec{n}$ is unit normal vector and $\vec{\tau}$ is the unit tangent vector, and $\frac{\partial}{\partial \tau}$ refers to the derivative of a scalar function in the direction of the tangent to $\Gamma$. 
Define the rigidity $K$ and average Poisson's ratio $\bar{\nu}$ by

$$
K=\overrightarrow{1}_{O}^{T} \mathbf{D}_{O} \mathbf{h}_{O}^{3} \overrightarrow{1}_{O}=\sum_{i \text { odd }} D_{i} h_{i}^{3}, \quad \bar{\nu}=\sum_{i \text { odd }} D_{i} h_{i}^{3} \nu_{i} / K
$$

One can check (since $D_{i}$ and $\nu_{i}$ are constant here) that

$$
\operatorname{div} L_{i} \nabla w=\Delta^{2} w, \quad\left(\overrightarrow{1}_{O}^{T} L_{O} \mathbf{D}_{O} \mathbf{h}_{O}^{3} \overrightarrow{1}_{O} \nabla w\right) \cdot \vec{n}=K(\Delta w)_{n} .
$$

Now let $\overline{\mathcal{B}}$ denote the boundary operator defined as $\mathcal{B}^{i}$ in (3.31) (any $i$ ) with $\nu_{i}$ replaced by $\bar{\nu}$. With this notation it follows that

$$
\begin{aligned}
\ell_{O}\left(\mathbf{h}_{O}^{3} \mathbf{D}_{O} \overrightarrow{1}_{O} \nabla w, \overrightarrow{1}_{O} \nabla \hat{w}\right)= & K\left[\left(\Delta^{2} w, \hat{w}\right)_{\Omega}+\left((\overline{\mathcal{B}} \nabla w) \cdot \vec{n}, \hat{w}_{n}\right)_{\Gamma_{1}}\right. \\
& \left.-\left(\frac{\partial}{\partial \tau}\{(\overline{\mathcal{B}} \nabla w) \cdot \vec{\tau}\}+(\Delta w)_{n}, \hat{w}\right)_{\Gamma_{1}}\right] .
\end{aligned}
$$

\subsection{Thin compliant layer model}

In the three-layer Mead-Markus model, the only energy accounted for in the compliant layer is the potential energy due to shear and the transverse kinetic energy. The same can be accomplished in the multilayer case by limiting to zero the coefficients $\mathbf{D}_{E}$ and $\mathbf{p}_{E}$ that appear in the forms $a_{E}$ and $c_{E}$ in (2.12). The resulting thin compliant layer Rao-Nakra retains the same existence and uniqueness properties that the multilayer Rao-Nakra models does since the limit model satisfies the hypothesis of Theorem 2.1. Moreover, by Theorem 2.2 we know that this perturbation is regular in the sense that solutions to VP1 vary continuously under this limit.

When $\mathbf{p}_{E} \rightarrow 0$ we have

$$
\begin{aligned}
c\left(v_{O}, w ; \hat{v}_{O}, \hat{w}\right) & =(\mathbf{h} \cdot \mathbf{p} w, \hat{w})_{\Omega}+\frac{1}{12}\left(\mathbf{p}_{O} \mathbf{h}_{O}^{3} \vec{\nabla}_{O} w, \vec{\nabla}_{O} \hat{w}\right)_{\Omega}+\left(\mathbf{h}_{O} \mathbf{p}_{O} v_{O}, \hat{v}_{O}\right)_{\Omega} \\
& =(m w, \hat{w})_{\Omega}+(\alpha \nabla w, \nabla \hat{w})_{\Omega}+\left(\mathbf{h}_{O} \mathbf{p}_{O} v_{O}, \hat{v}_{O}\right)_{\Omega}
\end{aligned}
$$

where

$$
m=\mathbf{h} \cdot \mathbf{p}=\sum_{i=1}^{n} h_{i} \rho_{i}, \quad \alpha=\frac{1}{12} \overrightarrow{1}_{O}^{T} \mathbf{p}_{O} \mathbf{h}_{O}^{3} \overrightarrow{1}_{O}=\frac{1}{12} \sum_{i \text { odd }}^{n} \rho_{i} h_{i}^{3} .
$$

In this case the forms $a$ and $c$ become:

$$
\begin{aligned}
a\left(v_{O}, w ; \hat{v}_{O}, \hat{w}\right) & =\ell_{O}\left(\mathbf{h}_{O}^{3} \mathbf{D}_{O} \overrightarrow{1}_{O} \nabla w, \overrightarrow{1}_{O} \nabla \hat{w}\right)+12 \ell_{O}\left(\mathbf{h}_{O} \mathbf{D}_{O} v_{O} ; \hat{v}_{O}\right)+\left(G_{E} \mathbf{h}_{E} \phi_{E}, \hat{\phi}_{E}\right)_{\Omega} \\
c\left(v_{O}, w ; \hat{v}_{O}, \hat{w}\right) & =(m w, \hat{w})_{\Omega}+(\alpha \nabla w, \nabla w)_{\Omega}+\left(\mathbf{h}_{O} \mathbf{p}_{O} v_{O}, \hat{v}_{O}\right)_{\Omega} .
\end{aligned}
$$

Note that the only potential energy remaining associated with the even layers is the shear energy term $\left(G_{E} \mathbf{h}_{E} \phi_{E}, \hat{\phi}_{E}\right)$.

The explicit formulation of the variational differential equation in (2.21) is

$$
\begin{aligned}
(m \ddot{w}, \hat{w})_{\Omega}+ & \alpha(\nabla \ddot{w}, \nabla \hat{w})_{\Omega}+\left(\mathbf{h}_{O} \mathbf{p}_{O} \ddot{v}_{O}, \hat{v}_{O}\right)_{\Omega}+\ell_{O}\left(\mathbf{h}_{O}^{3} \mathbf{D}_{O} \overrightarrow{1}_{O} \nabla w ; \overrightarrow{1_{O}} \nabla \hat{w}\right) \\
& +12 \ell_{O}\left(\mathbf{h}_{O} \mathbf{D}_{O} v_{O} ; \hat{v}_{O}\right)+\left(\mathbf{G}_{E} \mathbf{h}_{E} \varphi_{E}, \mathbf{h}_{E}^{-1} B \hat{v}_{O}+\vec{N} \nabla \hat{w}\right) \\
= & \int_{\Omega} \hat{w} f_{3}+\hat{v}_{O} \cdot f_{O} d x+\int_{\Gamma_{1}} \hat{w} g_{3}-\hat{w}_{n} M_{n}+\hat{v}_{O} \cdot g_{O} d s
\end{aligned}
$$


Using integrations by parts one obtains the following

$$
\left(E_{1}, \hat{w}\right)_{\Omega}+\left(E_{2}, \hat{v}_{O}\right)_{\Omega}+\left(E_{3}, \hat{w}\right)_{\Gamma_{1}}+\left(E_{4}, \hat{w}_{n}\right)_{\Gamma_{1}}+\left(E_{5}, \hat{v}_{O}\right)_{\Gamma_{1}}=0
$$

where under the assumptions that the $D_{i}, h_{i}$, and $\nu_{i}$ are constant in each layer,

$$
\begin{aligned}
E_{1} & =m \ddot{w}-\alpha \Delta \ddot{w}+K \Delta^{2} w-\operatorname{div} \vec{N}^{T} \mathbf{G}_{E} \mathbf{h}_{E} \varphi_{E}-f_{3} \\
E_{2} & =\mathbf{h}_{O} \mathbf{p}_{O} \ddot{v_{O}}-12 \mathbf{h}_{O} \mathbf{D}_{O} L_{O} v_{O}+B^{T} \mathbf{G}_{E} \varphi_{E}-f_{O} \\
E_{3} & =\alpha \ddot{w}_{n}-K\left(\frac{\partial}{\partial \tau}(\overline{\mathcal{B}} \nabla w) \cdot \vec{\tau}\right)-K(\Delta w)_{n}+\vec{N}^{T} \mathbf{G}_{E} \mathbf{h}_{E} \varphi_{E} \cdot \vec{n}-g_{3} \\
E_{4} & =K(\overline{\mathcal{B}} \nabla w) \cdot \vec{n}+M_{n} \quad E_{5}=12 \mathbf{D}_{O} \mathcal{B}_{O} \mathbf{h}_{O} v_{O}-g_{O} .
\end{aligned}
$$

In the above, $\frac{\partial}{\partial \tau}$ refers to the tangential derivative, while the subscript $n$ indicates a normal derivative.

The associated boundary value problem is

$$
\begin{aligned}
& \left.\begin{array}{rl}
m \ddot{w}-\alpha \Delta \ddot{w}+K \Delta^{2} w-\operatorname{div} \vec{N}^{T} \mathbf{G}_{E} \mathbf{h}_{E} \varphi_{E} & =f_{3} \\
\mathbf{h}_{O} \mathbf{p}_{O} \ddot{v}_{O}-12 \mathbf{h}_{O} \mathbf{D}_{O} L_{O} v_{O}+B^{T} \mathbf{G}_{E} \varphi_{E} & =f_{O}
\end{array}\right\} \quad \text { in } \Omega \times(0, \infty) \\
& \left.\begin{array}{rl}
\alpha \ddot{w}_{n}-K\left(\frac{\partial}{\partial \tau}(\overline{\mathcal{B}} \nabla w) \cdot \vec{\tau}\right)-K(\Delta w)_{n} & \\
+\vec{N}^{T} \mathbf{G}_{E} \mathbf{h}_{E} \varphi_{E} \cdot \vec{n} & =g_{3} \\
(\overline{\mathcal{B}} \nabla w) \cdot \vec{n}=-M_{n}, \quad 12 \mathbf{D}_{O} \mathcal{B}_{O} \mathbf{h}_{O} v_{O} & =g_{O}
\end{array}\right\} \quad \text { on } \Gamma_{1} \times(0, \infty) \\
& w=w_{n}=0, \quad v_{O}=0 \quad \text { on } \Gamma_{0} \times(0, \infty) .
\end{aligned}
$$

Appropriate initial conditions compatible with finite energy solutions are of the form

$$
\left\{v_{O}(0), w(0)\right\}=\left\{v_{O}^{0}, w^{0}\right\}, \quad\left\{\dot{v}_{O}(0), \dot{w}(0)\right\}=\left\{v_{O}^{1}, w^{1}\right\}
$$

From Theorem 2.1, the following holds:

Proposition 3.1 Given any $y^{0}=\left\{v_{O}^{0}, w^{0}\right\} \in \mathcal{V}$ and $y^{1}=\left\{v_{O}^{1}, w_{O}^{1}\right\} \in \mathcal{H}$ there exists a unique variational solution $y \in C([0, T], \mathcal{V}) \cap C^{1}([0, T], \mathcal{H})$ to (3.41)-(3.43).

It is also a consequence of Theorem 2.2 that the solution $\{y, \dot{y}\}$ is the limit in $L^{2}(0, T ; \mathcal{V} \times$ $\mathcal{H}$ ) of solutions to $\mathbf{V P 1}$ (with parameters $\mathbf{D}_{E}, \mathbf{p}_{E}$ ) as $\mathbf{D}_{E}, \mathbf{p}_{E}$ tend to zero in $\mathbf{R}^{m}$.

\subsubsection{Thin compliant layer model with shear damping}

To write out equations of motion with shear damping included one simply applies the correspondence

$$
\mathbf{G}_{E} \varphi_{E} \rightarrow\left(\mathbf{G}_{E} \varphi_{E}+\tilde{\mathbf{G}}_{E} \dot{\varphi}_{E}\right)
$$

The equations of motion become

$$
\begin{aligned}
& \left.\begin{array}{rll}
m \ddot{w}-\alpha \Delta \ddot{w}+K \Delta^{2} w-\operatorname{div} \vec{N}^{T}\left(\mathbf{G}_{E} \mathbf{h}_{E} \varphi_{E}+\tilde{\mathbf{G}}_{E} \mathbf{h}_{E} \dot{\varphi}_{E}\right) & =f_{3} \\
\mathbf{h}_{O} \mathbf{p}_{O} \ddot{v}_{O}-12 \mathbf{h}_{O} \mathbf{D}_{O} L_{O} v_{O}+B^{T}\left(\mathbf{G}_{E} \varphi_{E}+\tilde{\mathbf{G}}_{E} \dot{\varphi}_{E}\right) & =f_{O}
\end{array}\right\} \quad \text { in } \Omega \times(0, \infty) \\
& \alpha \ddot{w}_{n}-\left(\frac{\partial}{\partial \tau}(\vec{\tau} \cdot K \overline{\mathcal{B}} \nabla w)\right)-K(\Delta w)_{n} \\
& +\vec{N}^{T}\left(\mathbf{G}_{E} \mathbf{h}_{E} \varphi_{E}+\tilde{\mathbf{G}}_{E} \mathbf{h}_{E} \dot{\varphi}_{E}\right) \cdot \vec{n}=g_{3} \\
& K \overline{\mathcal{B}} \nabla w \cdot \vec{n}=-M_{n} \\
& 12 \mathbf{D}_{O} \mathcal{B}_{O} \mathbf{h}_{O} v_{O}=g_{O} \\
& v_{O}=0, \quad w=0, \quad w_{n}=0 \quad \text { on } \Gamma_{0} \times(0, \infty) \\
& \text { on } \Gamma_{1} \times(0, \infty)
\end{aligned}
$$


where $\varphi_{E}=\mathbf{h}_{E}^{-1} B v_{O}+\vec{N} \nabla w$.

It follows from Proposition 2.1 that the above damped system is well-posed on the same function space $C([0, T], \mathcal{V}) \cap C^{1}([0, T], \mathcal{H})$ as the undamped system (i.e., as in Proposition $3.1)$.

\subsection{Laminated plate with adhesive bonding}

In a laminated plate model, many layers of one or more materials may be bonded together by an adhesive. With many adhesive layers it becomes increasingly important to include the effect of shear from the adhesive layers. One possibility to model such a plate is to estimate the ratios of shear modulus to thickness $\left(\gamma_{i}:=G_{i} / h_{i}\right)$ in the compliant layers and then pass to the limit as the thicknesses and shear moduli of the compliant layers tend to zero in VP1 (or equivalent, in (3.41)-(3.43)) such that $\gamma_{i}, i$ even, are fixed. Therefore define

$$
\gamma=\mathbf{h}_{E}^{-1} \mathbf{G}_{E} .
$$

Also define

$$
s=B v_{O}+A \mathbf{h}_{O} \overrightarrow{1}_{O} \nabla w=: B v_{O}+\vec{M} \nabla w, \quad\left(\vec{M}=A \mathbf{h}_{O} \overrightarrow{1}_{O}\right) .
$$

Note that $s$ is independent of $\mathbf{h}_{E}$ and $\mathbf{G}_{E}$.

Theorem 2.2 will apply to the limit of $\mathbf{V P 1}$ as $\mathbf{h}_{E} \rightarrow 0, \mathbf{G}_{E} \rightarrow 0$ such that $\gamma$ is fixed provided the limiting variational forms $a$ and $c$ satisfy the appropriate estimates in Lemmas 2.1 and 2.2. To this end we have the following:

Lemma 3.1 Let $\mathbf{h}_{E}$ and $\mathbf{G}_{E}$ tend to 0 such that $\boldsymbol{\gamma}$ is fixed. Then for fixed $v_{O}, \hat{v}_{O}, w$, $\hat{w}$, with $s$ and $\hat{s}$ defined as in (3.47) the following holds:

$$
\begin{aligned}
& \left(\mathbf{G}_{E} \mathbf{h}_{E} \varphi_{E}, \hat{\varphi}_{E}\right)_{\Omega} \rightarrow(\boldsymbol{\gamma} s, \hat{s})_{\Omega} \quad \text { as } h \rightarrow 0, \\
& a_{E}\left(\psi_{E}, v_{E} ; \hat{\phi}_{E}, \hat{v}_{E}\right) \rightarrow 0 \quad \text { as } h \rightarrow 0 \text {, } \\
& c_{E}\left(\psi_{E}, v_{E} ; \hat{\phi}_{E}, \hat{v}_{E}\right) \rightarrow 0 \quad \text { as } h \rightarrow 0
\end{aligned}
$$

The forms $a_{O}$ and $c_{O}$ are unchanged by this limit.

Proof: In each case one simply expresses the forms in terms of the state variables and calculates the limit. To compute the first one,

$$
\begin{aligned}
\left(\mathbf{G}_{E} \mathbf{h}_{E} \varphi_{E}, \hat{\varphi}_{E}\right)_{\Omega} & =\left(\mathbf{G}_{E} \mathbf{h}_{E}\left(\mathbf{h}_{E}^{-1} B v_{O}+\vec{N} \nabla w\right), \mathbf{h}_{E}^{-1} B \hat{v}_{O}+\vec{N} \nabla \hat{w}\right)_{\Omega} \\
& =\left(\gamma\left(B v_{O}+\left(A \mathbf{h}_{O} \overrightarrow{1}_{O}+\mathbf{h}_{E} \overrightarrow{1}_{E}\right) \nabla w\right), B \hat{v}_{O}+\left(A \mathbf{h}_{O} \overrightarrow{1}_{O}+\mathbf{h}_{E} \overrightarrow{1}_{E}\right) \nabla \hat{w}\right)_{\Omega} \\
& \rightarrow(\gamma s, \hat{s})_{\Omega}
\end{aligned}
$$

The next two limits are computed the same way.

It follows that the limiting form of the equations of motion can be obtained from (3.41)(3.43) from the correspondence:

$$
\begin{aligned}
\mathbf{G}_{E} \mathbf{h}_{E} & \rightarrow \gamma \\
\varphi_{E} & \rightarrow s \\
\mathbf{h}_{E}^{-1} B & \rightarrow B \\
\vec{N} & \rightarrow \vec{M}:=A \mathbf{h}_{O} \overrightarrow{1}_{O}
\end{aligned}
$$


One then obtains the following laminated plate system

$$
\begin{aligned}
& \left.\begin{array}{l}
m \ddot{w}-\alpha \Delta \ddot{w}+K \Delta^{2} w-\operatorname{div} \vec{M}^{T} \boldsymbol{\gamma} s=f_{3} \\
\mathbf{h}_{O} \mathbf{p}_{O} \ddot{v}_{O}-12 \mathbf{h}_{O} \mathbf{D}_{O} L_{O} v_{O}+B^{T} \boldsymbol{\gamma} s=f_{O}
\end{array}\right\} \quad \text { on } \Omega \times(0, \infty) \\
& \left.\begin{array}{rl}
\alpha \ddot{w}_{n}-\frac{\partial}{\partial \tau}(\vec{\tau} \cdot K \overline{\mathcal{B}} \nabla w)-K(\Delta w)_{n}+\vec{M}^{T} \boldsymbol{\gamma} \cdot \vec{n} & =g_{3} \\
-K \overline{\mathcal{B}} \nabla w \cdot \vec{n} & =M_{n} \\
12 \mathbf{D}_{O} \mathcal{B}_{O} \mathbf{h}_{O} v_{O} & =g_{O}
\end{array}\right\} \quad \text { on } \Gamma_{1} \times(0, \infty) \\
& v_{O}=0, \quad w=0, \quad w_{n}=0 \quad \text { on } \Gamma_{0} \times(0, \infty),
\end{aligned}
$$

where $s$ is given in (3.47).

It is easy to check, using Lemma 3.1 that the limiting forms $a$ and $c$ satisfy the estimates in Lemmas 2.1 and 2.2 and consequently Theorems 2.1 and 2.2 remain valid for limiting system.

Proposition 3.2 Given any $y^{0}=\left\{v_{O}^{0}, w^{0}\right\} \in \mathcal{V}$ and any $y^{1}=\left\{v_{O}^{1}, w^{1}\right\} \in \mathcal{H}$ there exists a unique variational solution $y=\left\{v_{O}, w\right\} \in C([0, T], \mathcal{V}) \cap C^{1}([0, T], \mathcal{H})$ to (3.49)-(3.51) such that $y(0)=y^{0}, \dot{y}(0)=y^{1}$.

It is also worth noting (since we have verified that the result of Theorem 2.2 applies) that solutions to (3.49)-(3.51) can be obtained as the limit as $\mathbf{h}_{E} \rightarrow 0, \mathbf{G}_{E} \rightarrow 0$ with $\boldsymbol{\gamma}$ fixed of VP1 (or to (3.49)-(3.51)) in the space $L^{2}((0, T), \mathcal{V} \times \mathcal{H})$, as is described in Theorem 2.2.

Remark 3.1 To include shear damping in the laminated plate model (3.49)-(3.51), simply apply the correspondence

$$
\gamma s \rightarrow(\gamma s+\tilde{\gamma} \dot{s})
$$

The diagonal matrix $\tilde{\gamma}$ is defined analogous to the way $\boldsymbol{\gamma}$ was defined, i.e., $\tilde{\boldsymbol{\gamma}}=\tilde{\mathbf{G}}_{E} \mathbf{h}_{E}^{-1}$ in the damped thin compliant layer model (Section 3.2.1) and the damped laminated plate model is obtained by passing to the limit as $\mathbf{h}_{E} \rightarrow 0, \mathbf{G}_{E} \rightarrow 0$ and $\tilde{\mathbf{G}}_{E} \rightarrow 0$ with $\boldsymbol{\gamma}$ and $\tilde{\boldsymbol{\gamma}}$ fixed.

Since

$$
(\tilde{\gamma} s, s)_{\Omega}=\left(\tilde{\gamma}\left(B v_{O}+\vec{M} \nabla w\right), B v_{O}+\vec{M} \nabla w\right)_{\Omega} \leq C\left\|\left\{v_{O}, w\right\}\right\|_{\mathcal{H}}^{2}
$$

Proposition 2.1 applies to damped system. Hence the damped system (i.e., (3.49)-(3.51) with the correspondence (3.52)) has the same existence and uniqueness properties described in Proposition 3.2 for the undamped system.

\section{Multilayer Mead-Markus model}

In the three-layer Mead-Markus model, the in-plane and rotational inertias of all layers are ignored (along with the bending stresses in the core layer). An analogous multilayer model which we call the "multilayer Mead-Markus model" can be obtained by dropping the dynamic terms $\ddot{v}_{O},-\alpha \Delta \ddot{w}$ in the thin compliant layer model (3.41)-(3.43). This type of perturbation is singular, and hence one would not expect solutions to behave continuously with respect to this perturbation. Nevertheless dispersion relations (Rao, Nakra [18]) and eigenvalue investigations (Hansen, Spies [6]) indicate that this type of approximation provides a close approximation to the original (three-layer) Rao-Nakra system in the low-frequency range. 
In order to allow slightly more generality, here we include the rotational inertia term $-\alpha \Delta \ddot{w}$, with the understanding that $\alpha \geq 0$, with $\alpha=0$ corresponding to the classical Mead-Markus model. The boundary value problem for the multilayer Mead-Markus model is

$$
\begin{aligned}
& \left.\begin{array}{rl}
m \ddot{w}-\alpha \Delta \ddot{w}+K \Delta^{2} w-\operatorname{div} \vec{N}^{T} \mathbf{G}_{E} \mathbf{h}_{E} \varphi_{E} & =f_{3} \\
-12 \mathbf{h}_{O} \mathbf{D}_{O} L_{O} v_{O}+B^{T} \mathbf{G}_{E} \varphi_{E} & =f_{O}
\end{array}\right\} \quad \text { in } \Omega \times(0, \infty) \\
& \left.\begin{array}{rl}
\alpha \ddot{w}_{n}-K\left(\frac{\partial}{\partial \tau}(\vec{\tau} \cdot \overline{\mathcal{B}} \nabla w)+(\Delta w)_{n}\right) & \\
+\vec{N}^{T} \mathbf{G}_{E} \mathbf{h}_{E} \varphi_{E} \cdot \vec{n} & =g_{3} \\
K \overline{\mathcal{B}} \nabla w \cdot \vec{n} & =-M_{n} \\
12 \mathbf{D}_{O} \mathcal{B}_{O} \mathbf{h}_{O} v_{O} & =g_{O}
\end{array}\right\} \\
& v_{O}=0, \quad w=0, \quad w_{n}=0 \quad \text { on } \Gamma_{0} \times(0, \infty) \\
& \text { where } \mathbf{h}_{E} \varphi_{E}=B v_{O}+\mathbf{h}_{E} \vec{N} \nabla w
\end{aligned}
$$

Initial conditions are specified for $\{w, \dot{w}\}$.

Define

$$
\mathcal{H}_{\alpha}= \begin{cases}L^{2}(\Omega) & \text { if } \alpha=0 \\ H_{\Gamma_{0}}^{1} & \text { if } \alpha>0\end{cases}
$$

The variational form of this problem can be written

VP2: Find $y=\left\{v_{O}, w\right\}$ such that

$$
\begin{aligned}
& y \in C([0, T], \mathcal{V}), \quad \dot{w} \in C\left([0, T], \mathcal{H}_{\alpha}\right) \\
& m(\ddot{w}, \hat{w})_{\Omega}+\alpha(\nabla \ddot{w}, \nabla \hat{w})_{\Omega}+K \ell_{O}(\nabla w ; \nabla \hat{w})+\left(\mathbf{G}_{E} \mathbf{h}_{E} \varphi_{E}, \hat{\varphi}_{E}\right)_{\Omega} \\
& \quad+12 \ell_{O}\left(\mathbf{h}_{O} \mathbf{D}_{O} v_{O} ; \hat{v}_{O}\right)=\mathcal{W}(\hat{y}) \quad \forall \hat{y}=\left\{\hat{v}_{O}, \hat{w}\right\} \in \mathcal{V}
\end{aligned}
$$

(in the sense of distributions on $(0, T)$ )

$$
\left.\left\{w, w_{t}\right\}\right|_{t=0}=\left\{w^{0}, w^{1}\right\} \quad \text { given in } H_{\Gamma_{0}}^{2} \times \mathcal{H}_{\alpha} .
$$

In (4.58), $\varphi_{E}$ is given by (4.56) and $\hat{\varphi}_{E}$ is related to $\hat{w}, \hat{v}_{O}$ by the same equation. All forces included in $\mathcal{W}$ are assumed to have the same regularity as described in VP1. Here however, we take $m, K$ to be positive constants, the diagonal elements of $\mathbf{h}_{O}, \mathbf{D}_{O}, \mathbf{h}_{E}$ are positive constants, $\alpha$ is a nonnegative constant, and the diagonal elements of $\mathbf{G}_{E}$ are nonnegative and continuous on $\bar{\Omega}$.

Theorem 4.1 There is a unique solution $y=\left\{v_{O}, w\right\}$ to VP2.

Actually, we will first solve for $v_{O}$ in terms of $\nabla w$ and obtain an equivalent variational problem.

The boundary value problem

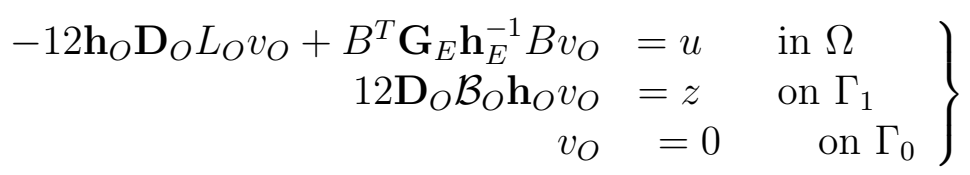

is easily seen to be associated with the variational equation

$$
12 \ell_{O}\left(\mathbf{h}_{O} \mathbf{D}_{O} v_{O} ; \hat{v}_{O}\right)+\left(\mathbf{G}_{E} \mathbf{h}_{E}^{-1} B v_{O}, B \hat{v}_{O}\right)_{\Omega}=\left(u, \hat{v}_{O}\right)_{\Omega}+\left(z, \hat{v}_{O}\right)_{\Gamma_{1}} \quad \forall \hat{v}_{O} \in H_{O, \Gamma_{0}}^{1} .
$$


The matrix $\mathbf{G}_{E} \mathbf{h}_{E}^{-1}$ is diagonal and positive and hence the term $\left(\mathbf{G}_{E} \mathbf{h}_{E}^{-1} B v_{O}, B \hat{v}_{O}\right)_{\Omega}$ in (4.61) does not alter the continuity or positivity of the form $\ell_{O}$. It hence follows from the coercive estimates in Lemma 2.1 that (4.61), and hence also (4.60) has a unique solution in $H_{O, \Gamma_{0}}^{1}$ whenever $u \in L_{O}^{2}(\Omega)$ and $z \in L_{O}^{2}\left(\Gamma_{1}\right)$. By superposition there are continuous operators $T_{1}: L_{O}^{2}(\Omega) \rightarrow H_{O, \Gamma_{0}}^{1}(\Omega)$ and $T_{2}: L_{O}^{2}\left(\Gamma_{1}\right) \rightarrow H_{O, \Gamma_{0}}^{1}(\Omega)$ such that

$$
v_{O}=T_{1} u+T_{2} z
$$

Define $J:\left(L^{2}(\Omega)\right)^{2} \rightarrow\left(H_{\Gamma_{0}}^{1}\right)^{2}$ by

$$
J=\vec{N}^{T} \mathbf{G}_{E} B T_{1} B^{T} \mathbf{G}_{E} \vec{N} .
$$

Using these operators the shear term in the first equation in (4.53) becomes

$$
\begin{aligned}
-\operatorname{div} \vec{N}^{T} \mathbf{G}_{E} \mathbf{h}_{E} \varphi_{E} & =-\operatorname{div} \vec{N}^{T} \mathbf{G}_{E}\left(B v_{O}+\mathbf{h}_{E} \vec{N} \nabla w\right) \\
& =-\vec{N}^{T} \mathbf{G}_{E} \mathbf{h}_{E} \vec{N} \Delta w-\operatorname{div} \vec{N}^{T} \mathbf{G}_{E} B\left(T_{1}\left(f_{O}-B^{T} \mathbf{G}_{E} \vec{N} \nabla w\right)+T_{2} g_{O}\right) \\
& =-\vec{N}^{T} \mathbf{G}_{E} \mathbf{h}_{E} \vec{N} \Delta w+\operatorname{div} J \nabla w-\operatorname{div} \vec{N}^{T} \mathbf{G}_{E} B\left(T_{1} f_{O}+T_{2} g_{O}\right) .
\end{aligned}
$$

Therefore the first equation in (4.53) becomes

$$
\begin{array}{r}
m \ddot{w}-\alpha \Delta \ddot{w}+K \Delta^{2} w-\vec{N}^{T} \mathbf{G}_{E} \mathbf{h}_{E} \vec{N} \Delta w+\operatorname{div} J \nabla w=\tilde{f}_{3} \quad \text { in } \Omega \times(0, \infty) \\
\text { where } \quad \tilde{f}_{3}=f_{3}+\operatorname{div} \vec{N}^{T} \mathbf{G}_{E} \mathbf{h}_{E}^{-1} B\left(T_{1} f_{O}+T_{2} g_{O}\right) .
\end{array}
$$

Similarly the boundary conditions for $w$ are easily obtained:

$$
\begin{aligned}
\alpha \ddot{w}_{n}-K \frac{\partial}{\partial \tau}[(\overline{\mathcal{B}} \nabla w) \cdot \tau]-K(\Delta w)_{n} & \\
-(J \nabla w) \cdot n+\vec{N}^{T} \mathbf{G}_{E} \mathbf{h}_{E} \vec{N} w_{n} & =\tilde{g}_{3} \quad \text { on } \Gamma_{1} \times(0, \infty) \\
\left(\text { where } \tilde{g}_{3}=g_{3}-\vec{N}^{T} \mathbf{G}_{E} B\left(T_{1} f_{O}+T_{2} g_{O}\right) \cdot \vec{n}\right) & \\
-K(\overline{\mathcal{B}} \nabla w) \cdot \vec{n} & =M_{n} \quad \text { on } \Gamma_{1} \times(0, \infty) \\
w=w_{n} & =0 \quad \text { on } \Gamma_{0} \times(0, \infty) .
\end{aligned}
$$

Let us see that the above system corresponds to a well-posed variational problem.

We multiply (4.64) by $\hat{w} \in H_{\Gamma_{0}}^{2}$ and obtain after integrations by parts

$$
\begin{array}{r}
(m \ddot{w}, \hat{w})_{\Omega}+\alpha(\nabla \ddot{w}, \nabla \hat{w})_{\Omega}+K \ell_{O}(\nabla w ; \hat{\nabla} w)+\vec{N}^{T} \mathbf{G}_{E} \mathbf{h}_{E} \vec{N}(\nabla w, \nabla \hat{w})_{\Omega} \\
-(J \nabla w, \nabla \hat{w})_{\Omega}=\left(\tilde{f}_{3}, \hat{w}\right)_{\Omega}+\left(\tilde{g}_{3}, \hat{w}\right)_{\Gamma_{1}} \quad \forall \hat{w} \in H_{\Gamma_{0}}^{2}
\end{array}
$$

Thus VP2 has been reduced to the following problem.

VP2.1: Find $\{w\}$ such that

$$
\begin{aligned}
& w \in C\left([0, T], H_{\Gamma_{0}}^{2}\right), \quad \dot{w} \in C\left([0, T], \mathcal{H}_{\alpha}\right) \\
& (4.68) \text { holds (in the sense of distributions on }(0, T)) \\
& \left.w\right|_{t=0}=w^{0} \text { given in } H_{\Gamma_{0}}^{2},\left.\quad \dot{w}\right|_{t=0}=w^{1} \text { given in } \mathcal{H}_{\alpha}
\end{aligned}
$$


Proposition 4.1 There exists a unique solution $w$ that solves VP2.1. Moreover, $y:=$ $\left\{v_{O}, w\right\}$, with $v_{O}=T_{1}\left(f_{O}-B^{T} \mathbf{G}_{E} \vec{N} \nabla w\right)+T_{2}\left(g_{O}\right)$ is the unique solution to VP2.

Proof: First, let us see that VP2.1 is well set. Since we already have from Lemma 2.1 that the form $a(w ; \hat{w}):=\ell_{O}(\nabla w, \nabla w)$ is continuous, symmetric and coercive on $H_{\Gamma_{0}}^{2}$, and the form $c(w ; \hat{w}):=(m w, \hat{w})_{\Omega}+(\alpha \nabla w, \nabla \hat{w})_{\Omega}$ is continuous, symmetric and coercive on $\mathcal{H}_{\alpha}$ (the case $\alpha=0$ is trivial) it is enough to show that the form determined by the remaining terms in (4.68) are symmetric, nonnegative and continuous on $H_{\Gamma_{0}}^{2}$. To this end let $q(w, \hat{w})=$ $\overrightarrow{N^{T}} \mathbf{G}_{E} \mathbf{h}_{E} \vec{N}(\nabla w, \hat{\nabla} w)_{\Omega}-(J \nabla w, \nabla \hat{w})_{\Omega}$. Let $r=-T_{1} B^{T} \mathbf{G}_{E} \vec{N} \nabla w$ (so $r$ plays the role of $v_{O}$ ) and likewise define $\hat{r}$ in terms of $\nabla \hat{w}$. Let $s=\mathbf{h}_{E}^{-1} B r+\vec{N} \nabla w$ (so $s$ plays the role of $\varphi_{E}$ ) and likewise define $\hat{s}$ in terms of $\hat{w}$ and $\hat{r}$. Then

$$
\begin{aligned}
q(w, \hat{w}) & =\left(\mathbf{G}_{E} \mathbf{h}_{E} \vec{N} \nabla w, \vec{N} \nabla \hat{w}\right)_{\Omega}-\left(\mathbf{G}_{E} B T_{1} B^{T} \mathbf{G}_{E} \vec{N} \nabla w, \vec{N} \nabla \hat{w}\right)_{\Omega} \\
& =\left(\mathbf{G}_{E} \mathbf{h}_{E} s, \vec{N} \nabla \hat{w}\right)_{\Omega} \\
& =\left(\mathbf{G}_{E} \mathbf{h}_{E} s, \hat{s}\right)_{\Omega}-\left(\mathbf{G}_{E} s, B \hat{r}\right)_{\Omega} \\
& =\left(\mathbf{G}_{E} \mathbf{h}_{E} s, \hat{s}\right)_{\Omega}-\left(\mathbf{G}_{E} \mathbf{h}_{E}^{-1} B r, B \hat{r}\right)_{\Omega}-\left(B^{T} \mathbf{G}_{E} \vec{N} \nabla w, \hat{r}\right) \\
& =\left(\mathbf{G}_{E} \mathbf{h}_{E} s, \hat{s}\right)_{\Omega}+12 \ell_{O}\left(\mathbf{h}_{O} \mathbf{D}_{O} r ; \hat{r}\right)
\end{aligned}
$$

where the last line was obtained using (4.61) and the definition of $T_{1} u$ in (4.62) with $u=$ $-B^{T} \mathbf{G}_{E} \vec{N} \nabla w$ and $z=0$.

Thus $q$ is symmetric and nonnegative. Furthermore, using the continuity of $\ell_{O}$ on $\left(H_{\Gamma_{o}}^{1}\right)^{2}$ and of $T_{1}$ from $L_{O}^{2}(\Omega)$ to $H_{\Gamma_{0}}^{1}$, one easily sees that $q$ is continuous on $H_{\Gamma_{0}}^{2}$ : Since

$$
\begin{aligned}
\|r\|_{H_{O}^{1} \Gamma_{0}} & \leq C\|\nabla w\|_{H_{\Gamma_{0}}^{1}} \leq C\|w\|_{H_{\Gamma_{0}}^{2}} \\
\|r\|_{L_{O}^{2}} & \leq C\|\nabla w\|_{H_{\Gamma_{0}}^{1}} \leq C\|w\|_{H_{\Gamma_{0}}^{2}} \\
\|s\|_{L_{E}^{2}} & \leq C\left(\|r\|_{L_{O}^{2}}+\|\nabla w\|_{L^{2}(\Omega)}\right) \leq C\|w\|_{H_{\Gamma_{0}}^{2}}
\end{aligned}
$$

it follows that

$$
\begin{aligned}
q(w, w) & \leq C\left(\|s\|_{L_{E}^{2}}^{2}+\|r\|_{H_{O, \Gamma_{0}}^{1}}^{2}\right) \\
& \leq C\left(\|r\|_{L_{O}^{2}}+\|\nabla w\|_{L^{2}(\Omega)}\right)^{2}+\|\nabla w\|_{H_{\Gamma_{0}}^{1}}^{2} \\
& \leq C\|w\|_{H_{\Gamma_{0}}^{2}}^{2} .
\end{aligned}
$$

Hence the continuity requirement is satisfied. In addition it is easily checked using the definition of $\tilde{f}_{3}$ and $\tilde{g}_{3}$ in (4.64) and (4.65) that $\tilde{f}_{3}$ and $\tilde{g}_{3}$ are $L^{2}$ functions. Hence there is a unique solution $w$ to VP2.1.

Finally, the previous calculation shows that when external forces $f_{O}$ and $g_{O}$ vanish, the variational differential equations in VP2 and VP2.1 are equivalent. Essentially the same calculation but with $v_{O}=T_{1}\left(f_{O}-B^{T} \mathbf{G}_{E} \vec{N} \nabla w\right)+T_{2} g_{O}$ (and using the definition of $\tilde{f}_{3}$ and $\tilde{g}_{3}$ as in (4.64) and (4.65)) shows that one obtains the variational differential equation in VP2. The regularity and initial conditions in VP2 follow from those in VP2.1. This completes the proof. 
Remark 4.1 In the same way the laminated plate model (3.49)-(3.51) was obtained from VP1 by letting $\mathbf{G}_{E}$ and $\mathbf{h}_{E}$ tend to zero at constant ratio, one can obtain a laminated MeadMarkus model with adhesive bonding from VP2 or VP2.1 by the same limit. Again, the equations of motion are easily obtained from (4.53)-(4.56) by the correspondence in (3.48). One obtains the system

$$
\begin{aligned}
& m \ddot{w}-\alpha \Delta \ddot{w}+K \Delta^{2} w-\operatorname{div} \vec{M}^{T} \gamma s=f_{3} \quad \text { on } \Omega \times(0, \infty) \\
& -12 \mathbf{h}_{O} \mathbf{D}_{O} L_{O} v_{O}+B^{T} \boldsymbol{\gamma} s=f_{O} \text { on } \Omega \times(0, \infty) \\
& \alpha \ddot{w}_{n}-\left(\frac{\partial}{\partial \tau}(\vec{\tau} \cdot K \overline{\mathcal{B}} \nabla w)\right)-K(\Delta w)_{n}+\vec{M}^{T}(\boldsymbol{\gamma} s) \cdot \vec{n}=g_{3} \text { on } \Gamma_{1} \times(0, \infty) \\
& -K \overline{\mathcal{B}} \nabla w \cdot \vec{n}=M_{n} \text { on } \Gamma_{1} \times(0, \infty) \\
& 12 \mathbf{D}_{O} \mathcal{B}_{O} \mathbf{h}_{O} v_{O}=g_{O} \text { on } \Gamma_{1} \times(0, \infty) \\
& v_{O}=0, \quad w=0, \quad w_{n}=0 \quad \text { on } \Gamma_{0} \times(0, \infty),
\end{aligned}
$$

where $s$ is defined by (3.47). Initial conditions are specified for $w, \dot{w}$.

All the steps described in this section for the multilayer Mead-Markus model remain valid for the above model and hence the same existence and uniqueness properties described in Theorem 4.1 and Proposition 4.1 remain valid for the above system.

Remark 4.2: Damping can be included in the multilayer Mead-Markus model (or the laminated model of the previous remark) as discussed in Section 3. In particular, the multilayer Mead-Markus model with shear damping takes the form:

$$
\begin{aligned}
& \left.\begin{array}{rl}
m \ddot{w}-\alpha \Delta \ddot{w}+K \Delta^{2} w-\operatorname{div} \vec{N}^{T} \mathbf{h}_{E}\left(\mathbf{G}_{E} \varphi_{E}+\tilde{\mathbf{G}}_{E} \dot{\varphi}_{E}\right) & =f_{3} \\
-12 \mathbf{h}_{O} \mathbf{D}_{O} L_{O} v_{O}+B^{T}\left(\mathbf{G}_{E} \varphi_{E}+\tilde{\mathbf{G}}_{E} \dot{\varphi}_{E}\right) & =f_{O}
\end{array}\right\} \quad \text { in } \Omega \times(0, \infty) \\
& \left.\begin{array}{rl}
\alpha \ddot{w}_{n}-K\left(\frac{\partial}{\partial \tau}(\vec{\tau} \cdot \overline{\mathcal{B}} \nabla w)+(\Delta w)_{n}\right) & \\
+\vec{N}^{T} \mathbf{h}_{E}\left(\mathbf{G}_{E} \varphi_{E}+\tilde{\mathbf{G}}_{E} \dot{\varphi}_{E}\right) \cdot \vec{n} & =g_{3} \\
K \overline{\mathcal{B}} \nabla w \cdot \vec{n} & =-M_{n} \\
12 \mathbf{D}_{O} \mathcal{B}_{O} \mathbf{h}_{O} v_{O} & =g_{O}
\end{array}\right\} \quad \text { on } \Gamma_{1} \times(0, \infty) \\
& v_{O}=0, \quad w=0, \quad w_{n}=0 \quad \text { on } \Gamma_{0} \times(0, \infty) \\
& \text { where } \mathbf{h}_{E} \varphi_{E}=B v_{O}+\mathbf{h}_{E} \vec{N} \nabla w
\end{aligned}
$$

In this case initial conditions are specified for $w, \dot{w}$ and $v_{O}$.

Existence and uniqueness, and stability of the analogous damped beam model for various specialized cases has been studied in several papers: [5], [6], [7].

\section{$5 \quad$ Examples}

In order to review the various models and notation, two of the plate systems of this paper are written out in detail for the case of a three layer plate.

\subsection{Thin compliant layer model}

First we write out the thin compliant layer model (3.41) for the case of three layers $(n=3)$. The various matrix quantities involved are

$$
A=\left(\begin{array}{cc}
\frac{1}{2} & \frac{1}{2}
\end{array}\right), \quad B=\left(\begin{array}{cc}
-1 & 1
\end{array}\right), \quad \mathbf{h}_{O}=\operatorname{diag}\left(h_{1}, h_{3}\right), \quad \mathbf{h}_{E}=h_{2},
$$




$$
\begin{gathered}
\overrightarrow{1}_{O}=\left(\begin{array}{c}
1 \\
1
\end{array}\right), \quad \overrightarrow{1}_{E}=1, \quad \vec{N}=\mathbf{h}_{E}^{-1} A \mathbf{h}_{O} \overrightarrow{1}_{O}+\overrightarrow{1}_{E}=\frac{h_{1}+2 h_{2}+h_{3}}{2 h_{2}} \\
\mathbf{D}_{O}=\operatorname{diag}\left(D_{1}, D_{3}\right), \quad \mathbf{p}_{O}=\operatorname{diag}\left(\rho_{1}, \rho_{3}\right), \quad \mathbf{G}_{E}=G_{2}
\end{gathered}
$$

Other parameters appearing are

$$
\begin{gathered}
m=\rho_{1} h_{1}+\rho_{2} h_{2}+\rho_{3} h_{3}, \quad \alpha=\left(\rho_{1} h_{1}^{3}+\rho_{3} h_{3}^{3}\right) / 12, \quad \mathbf{G}_{E} \mathbf{h}_{E}=G_{2} h_{2}, \\
K=D_{1} h_{1}^{3}+D_{3} h_{3}^{3}, \quad \bar{\nu}=\left(\nu_{1} h_{1}^{3} D_{1}+\nu_{3} h_{3}^{3} D_{3}\right) / K
\end{gathered}
$$

The state variables are $w$ and $v_{O}$, where $v_{O}=\left(\begin{array}{c}v^{1} \\ v^{2}\end{array}\right)=\left(\begin{array}{cc}v_{1}^{1} & v_{2}^{1} \\ v_{1}^{2} & v_{1}^{2}\end{array}\right)$. The variable $\varphi_{E}=$ $\left(\begin{array}{ll}\varphi_{1}^{2} & \varphi_{2}^{2}\end{array}\right)$ is defined in terms of the state variables by

$$
\begin{aligned}
\varphi_{E} & =h_{E}^{-1} B v_{O}+\vec{N} \nabla w ; \\
& =\frac{v^{3}-v^{1}}{h_{2}}+\frac{h_{1}+2 h_{2}+h_{3}}{2 h_{2}} \nabla w ; \quad \nabla w=\left(\begin{array}{ll}
\frac{\partial w}{\partial x_{1}} & \frac{\partial w}{\partial x_{2}}
\end{array}\right) .
\end{aligned}
$$

The operators $L_{O}$ and $\mathcal{B}_{O}$ defined by

$$
L_{O} v_{O}=\left(\begin{array}{cc}
L_{1}^{1} v^{1} & L_{2}^{1} v^{1} \\
L_{1}^{3} v^{3} & L_{2}^{3} v^{3}
\end{array}\right), \quad \mathcal{B}_{O} v_{O}=\left(\begin{array}{cc}
\mathcal{B}_{1}^{1} v^{1} & \mathcal{B}_{2}^{1} v^{1} \\
\mathcal{B}_{1}^{3} v^{3} & \mathcal{B}_{2}^{3} v^{3}
\end{array}\right)
$$

where the $L_{j}^{i}$ and $\mathcal{B}_{j}^{i}$ operators are given explicitly in (3.30), (3.31).

Thus, the equations of motion on $\Omega \times(0, \infty)$ become

$$
\begin{array}{cc}
m \ddot{w}-\alpha \Delta \ddot{w}+K \Delta^{2} w-\frac{1}{2}\left(h_{1}+2 h_{2}+h_{3}\right) G_{2} \operatorname{div} \varphi^{2}= & f_{3} \\
h_{1} \rho_{1}\left\{\ddot{v}_{1}^{1}, \ddot{v}_{2}^{1}\right\}-12 h_{1} D_{1}\left\{L_{1}^{1} v^{1}, L_{2}^{1} v^{1}\right\}-G_{2}\left\{\varphi_{1}^{2}, \varphi_{2}^{2}\right\} & =\left\{f_{1}^{1}, f_{2}^{1}\right\} \\
h_{3} \rho_{3}\left\{\ddot{v}_{1}^{3}, \ddot{v}_{2}^{3}\right\}-12 h_{3} D_{3}\left\{L_{1}^{3} v^{3}, L_{2}^{3} v^{3}\right\}+G_{2}\left\{\varphi_{1}^{2}, \varphi_{2}^{2}\right\} & =\left\{f_{1}^{3}, f_{2}^{3}\right\} .
\end{array}
$$

The boundary conditions (3.42), (3.43) can be used, or others are easily obtained. For example, in the case of pinned boundary conditions with applied moments on all of $\Gamma$, the boundary conditions are

$$
-K w_{n n}=M_{n}, \quad w=0 \quad v_{O}=0, \quad \text { on } \Gamma \times(0, \infty)
$$

where $w_{n n}$ is the second derivative in the outward normal direction along $\Gamma$.

\subsection{Laminated plate model with damping in adhesive layers}

Next we write out the damped laminated plate model of (3.49)-(3.50) in the case of shear damping, again for the case of three layers.

First recall that the damped laminated plate model can be obtained from the damped thin compliant layer model of Section 3.2.1 by letting $\mathbf{G}_{E}, \tilde{\mathbf{G}}_{E}$ and $\mathbf{h}_{E}$ tend to zero in such a way that $\boldsymbol{\gamma}=\mathbf{h}_{E}^{-1} \mathbf{G}_{E}$ and $\tilde{\gamma}=\mathbf{h}_{E}^{-1} \tilde{\mathbf{G}}_{E}$ are fixed. In the three layer case, the matrices $\boldsymbol{\gamma}$ 
and $\tilde{\gamma}$ reduce to the scalars $\gamma_{2}$ and $\tilde{\gamma}_{2}$, respectively. The variable $s=s^{2}=\left\{s_{1}^{2}, s_{2}^{2}\right\}$ is defined in terms of the state variables $v_{O}, w$ by

$$
s=B v_{O}+\vec{M} \nabla w=v^{3}-v^{1}+\frac{h_{1}+h_{3}}{2} \nabla w .
$$

The equations of motion on $\Omega \times(0, \infty)$ become

$$
\begin{array}{cc}
m \ddot{w}-\alpha \Delta \ddot{w}+K \Delta^{2} w-\frac{1}{2}\left(h_{1}+h_{3}\right) \operatorname{div}\left(\gamma_{2}\left\{s_{1}^{2} s_{2}^{2}\right\}+\tilde{\gamma}_{2}\left\{\dot{s}_{1}^{2}, \dot{s}_{2}^{2}\right\}\right)= & f_{3} \\
h_{1} \rho_{1}\left\{\ddot{v}_{1}^{1}, \ddot{v}_{2}^{1}\right\}-12 h_{1} D_{1}\left\{L_{1}^{1} v^{1}, L_{2}^{1} v^{1}\right\}-\gamma_{2}\left\{s_{1}^{2}, s_{2}^{2}\right\}-\tilde{\gamma}_{2}\left\{\dot{s}_{1}^{2}, \dot{s}_{2}^{2}\right\} & =\left\{f_{1}^{1}, f_{2}^{1}\right\} \\
h_{3} \rho_{3}\left\{\ddot{v}_{1}^{3}, \ddot{v}_{2}^{3}\right\}-12 h_{3} D_{3}\left\{L_{1}^{3} v^{3}, L_{1}^{3} v^{3}\right\}+\gamma_{2}\left\{s_{1}^{2}, s_{2}^{2}\right\}+\tilde{\gamma}_{2}\left\{\dot{s}_{1}^{2}, \dot{s}_{2}^{2}\right\} & =\left\{f_{1}^{3}, f_{2}^{3}\right\} .
\end{array}
$$

If the plate is clamped on $\Gamma_{0}$ and subject to general forces on $\Gamma_{1}$, as in (3.50), (3.51) (but for the damped case). Then

$$
\left\{v_{1}^{i}, v_{2}^{i}\right\}=0, i=1,3 ; \quad w=0, w_{n}=0 \quad \text { on } \Gamma_{0} \times(0, \infty) .
$$

The boundary operators involving $\overline{\mathcal{B}}$ used in (3.50) become (using $(x, y)$ for $\left(x_{1}, x_{2}\right)$ ):

$$
\begin{aligned}
(\overline{\mathcal{B}} \nabla w(x, y)) \cdot \vec{n} & =\bar{\nu} \Delta w+(1-\bar{\nu})\left(w_{x x} n_{1}^{2}+w_{y y} n_{2}^{2}\right)+2(1-\bar{\nu}) w_{x y} n_{1} n_{2} \\
(\overline{\mathcal{B}} \nabla w(x, y)) \cdot \vec{\tau} & \left.=(1-\bar{\nu})\left(n_{1}^{2}-n_{2}^{2}\right) w_{x y}+n_{1} n_{2}(1-\bar{\nu})\left(w_{y y}-w_{x x}\right)\right)
\end{aligned}
$$

where $\vec{n}=\left(n_{1}, n_{2}\right)$ is the outward unit normal to $\Gamma$ and $\vec{\tau}=\left(-n_{2}, n_{1}\right)$ is the unit tangent vector to $\Gamma$.

Suppose, for simplicity that $\Gamma_{1}$ is flat, e.g., so $\vec{n}=(1,0)^{T}$. Then (using that $\frac{\partial}{\partial n}=\frac{\partial}{\partial x}$ and $\frac{\partial}{\partial \tau}=\frac{\partial}{\partial y}$ ) the force boundary conditions (3.50) (with damping included) on $\Gamma_{1} \times(0, \infty)$ become:

$$
\begin{aligned}
& \alpha \ddot{w}_{x}-K(1-\bar{\nu}) w_{x y y}-K\left(w_{x x}+w_{y y}\right)_{x}+\frac{h_{1}+h_{3}}{2}\left(\gamma_{2} s_{1}^{2}+\tilde{\gamma}_{2} \dot{s}_{1}^{2}\right)= g_{3} \\
&-K\left(w_{x x}+\bar{\nu} w_{y y}\right)= M_{n} \\
& 12 \mathbf{D}_{O} \mathcal{B}_{O} \mathbf{h}_{O} v_{O}=\begin{aligned}
&\left\{12 D_{1} h_{1}\left(\left(v_{1}^{1}\right)_{x}+\nu_{1}\left(v_{2}^{1}\right)_{y}\right), 12 D_{1} h_{1} \frac{1-\nu_{1}}{2}\left[\left(v_{2}^{1}\right)_{x}+\left(v_{1}^{1}\right)_{y}\right]\right\}=\left\{g_{1}^{1}, g_{2}^{1}\right\} \\
&\left\{12 D_{3} h_{3}\left(\left(v_{1}^{3}\right)_{x}+\nu_{3}\left(v_{2}^{3}\right)_{y}\right), 12 D_{3} h_{3} \frac{1-\nu_{3}}{2}\left[\left(v_{2}^{3}\right)_{x}+\left(v_{1}^{3}\right)_{y}\right]\right\}\left\{g_{1}^{3}, g_{2}^{3}\right\}
\end{aligned}
\end{aligned}
$$

\section{Acknowledgment}

This research was supported in part by the National Science Foundation under the grant DMS0205148.

The author also gratefully acknowledges useful technical suggestions from Oleg Imanuvilov of the Department of Mathematics of Iowa State University.

\section{References}

[1] R. Dautray and J.-L. Lions (with collaboration of M. Artola, M Cessenat, H. Lanchon), Mathematical Analysis and Numerical Methods for Science and Technology Vol. 5 Evolution Problems I, (Springer-Verlag, 1992). 
[2] R.A. DiTaranto Theory of vibratory bending for elastic and viscoelastic layered finite-length beams, J. Appl. Mech., 32, (1965) 881-886.

[3] A.E. Green and P.M. Naghdi A Theory of laminated composite plates. IMA J. Appl. Math. 29 (1982) 1-23

[4] S.W. Hansen A Dynamical model for multilayer plates with independent shear deformations Quart. Appl. Math. 55(4), (1997), 601-621.

[5] S.W. Hansen and I. Lasiecka Analyticity, hyperbolicity and uniform stability of semigroups arising in models of composite beams Math. Models Methods Appl. Sci. 10 (2000) no. 4, 555-580.

[6] S.W. Hansen and R.D. Spies Structural damping in laminated beam due to interfacial slip, J. Sound Vibr. 204 (1997), 183-202.

[7] S.W. Hansen and Z. Liu Analyticity of semigroup associated with laminated composite beam Control of Distributed Parameter and Stochastic Systems eds. S. Chen, X. Li, J. Yong, X.Y. Zhou pp 467-54, (Kluwer Acad. Publ., 1999).

[8] D.I.G. Jones, Handbook of Viscoelastic Vibration Damping, (Wiley, 2001)

[9] E.M. Kerwin Damping of flexural waves by a constrained viscoelastic layer J. of the Acoustical Society of America, 31(7) 952-962.

[10] R.K. Kapania and S. Raciti Recent advances in analysis of laminated beams and plates, Part I: Shear and Buckling AIAA Journal 27 No. 7, (1989), 923-934 31-38.

[11] J. Lagnese, Boundary Stabilization of Thin Plates, Vol. 10 of SIAM studies in applied mathematics, (Society for Industrial and Applied Mathematics, 1989).

[12] J.E. Lagnese and J.-L Lions Modelling, Analysis and Control of Thin Plates, collection: "Recherches en Mathématiques Appliquées" RMA 6, (Springer-Verlag, 1989).

[13] J. L. Lions and E. Magenes, Nonhomogeneous Boundary Value Problems and Applications, (Springer, 1972).

[14] D.J. Mead and S. Markus The forced vibration of a three-layer, damped sandwich beam with arbitrary boundary conditions J. Sound Vibr. 10 (1969) 163-175.

[15] R.D. Mindlin Influence of rotary inertia and shear on flexural motions of isotropic elastic plates J. Appl. Mech. 18 (1951), 31-38.

[16] A.K. Noor and W.S. Burton Assessment of shear deformation theories for multilayer composite plates, Appl. Mech. Rev. 42, no.1 (1989) 1-12

[17] A. Nosier and J.N. Reddy On vibration and buckling of symmetric laminated plates according to shear deformation theory Acta Mech. 94 (1992), 123-151.

[18] Y.V.K.S. Rao and B.C. Nakra Vibrations of unsymmetrical sandwich beams and plates with viscoelastic cores, J. Sound Vibr. 34(3) (1974) 309-326. 
[19] J.N. Reddy A generalization of two-dimensional theories of laminated composite plates, Comm. Appl Num. Meth. 3 (1987) 173-180

[20] E. Reissner On the bending of elastic plates, Q. Appl. Math. 4 (1947), 55-68.

[21] D. Ross, E.E. Ungar, and E.M. Kerwin Damping of plate flexural vibrations by means of viscoelastic laminae in J.E. Ruzika (ed.), Structural Damping, Sec. 3, (The American Society of Mechanical Engineers, 1959).

[22] C.T. Sun and Y.P. Lu Vibration Damping of Structural Elements, (Prentice Hall, 1995).

[23] M.-J. Yan and E.H. Dowell Governing equations for vibrating constrained-layer damping sandwich plates and beams J. Appl. Mech. 39 (1972), 1041-1046. 